\title{
Dynamic Pricing and Inventory Management with Regular and Expedited Supplies
}

\author{
Sean X. Zhou \\ Department of Decision Sciences and Managerial Economics, CUHK Business School, and Department of Systems Engineering and \\ Engineering Management, The Chinese University of Hong Kong, Shatin, N.T. Hong Kong, zhoux@se.cuhk.edu.hk \\ Xiuli Chao \\ Department of Industrial and Operations Engineering, University of Michigan, Ann Arbor, Michigan, USA, xchao@umich.edu
}

\begin{abstract}
$\mathrm{W}$ e consider a periodic-review inventory system with regular and expedited supply modes. The expedited supply is faster than the regular supply but incurs a higher cost. Demand for the product in each period is random and sensitive to its selling price. The firm determines its order quantity from each supply in each period as well as its selling price to maximize the expected total discounted profit over a finite or an infinite planning horizon. We show that, in each period if it is optimal to order from both supplies, the optimal inventory policy is determined by two state-independent thresholds, one for each supply mode, and a list price is set for the product; if only the regular supply is used, the optimal policy is a state-dependent base-stock policy, that is, the optimal base-stock level depends on the starting inventory level, and the optimal selling price is a markdown price that decreases with the starting inventory level. We further study the operational impact of such supply diversification and show that it increases the firm's expected profit, reduces the optimal safety-stock levels, and lowers the optimal selling price. Thus that diversification is beneficial to both the firm and its customers. Building upon these results, we conduct a numerical study to assess and compare the respective benefit of dynamic pricing and supply diversification.
\end{abstract}

Key words: optimal policy; pricing; inventory control; supply diversification; lead time

History: Received: August 2011; Accepted: December 2012 by Eric Johnson, after 2 revisions.

\section{Introduction}

Supply diversification and dynamic pricing are important strategic tools for companies to manage their supply and demand, respectively. Companies in various industries, for example, airlines and consumer electronics, have successfully adopted dynamic pricing to increase their profitability. For example, Dell sells its product through its website and offers promotion every week and even changes price daily. Maintaining two supply sources for one product/component is common in procurement practice. In particular, because cost and delivery lead time are two of the key supplier selection measures, firms often pair one responsive but costly supplier with a less responsive but also less costly supplier to meet fickle customer demand. For instance, many firms in the US fashion industry have moved their major manufacturing facilities offshore to take advantage of the lower production cost. However, some still prefer to maintain costly domestic facilities so that they can better respond to changes in market demand. With both supply diversification and dynamic pricing in place, an important question is how to jointly determine the optimal inventory replenishment and pricing strategies.

In this study, we address this issue by considering a periodic-review inventory model with two supply sources. One supplier provides a shorter delivery lead time but incurs a higher unit cost while the other one has a longer delivery lead time but a lower unit cost. Demand is stochastic and sensitive to the selling price. Unsatisfied demand is fully backlogged in each period. Our objective is to determine the optimal inventory replenishment and pricing strategies that maximize the expected total discounted profit.

Inventory models with multiple supplies differing in cost and lead time have been extensively studied. Barankin (1961) is considered as the earliest work on inventory models with two delivery modes that studies a single period problem. Daniel (1963) extends Barankin (1961) to a multi-period model with one capacitated regular supplier and one emergency supplier, with lead times being 1 and 0 , respectively. Fukuda (1964) further generalizes the work of Daniel (1961) to the case where the lead times of the two supply modes are $L$ (an arbitrary non-negative constant) and $L+1$, respectively. The dual-supplier problem with arbitrary length of lead times is studied by 
Whittmore and Saunders (1977), who demonstrate that the optimal control policy is very complicated and state dependent if the difference in lead times is not 1 . That is, the optimal policy control parameters are functions of the state of the system. Because of the complexity of the optimal policy, recently, SchellerWolf et al. (2003) and Veeraraghavan and SchellerWolf (2008) focused on evaluation and optimization of two classes of inventory policies, that is, "single index" and "dual index" policies, respectively. Allon and Van Mieghem (2010) develop a continuous-time model and investigate the average order rates placed to each source when two supply sources are available with different costs and responsiveness. Other related work on single-stage inventory systems with multiple transportation modes includes Feng et al. (2003, 2004).

Another closely related stream of research is the coordination of inventory replenishment and pricing. This topic, starting with the work of Whitin (1955), who analyzes the newsvendor problem with pricedependent demand, has been the focus of many papers. Federgruen and Heching (1999) characterize the optimal inventory and pricing policy as a basestock list-price policy for both finite and infinite planning horizon problems. By list-price policy, we mean that the selling price is usually set at a constant price, referred to as list price, but as the inventory level gets higher, the firm offers a discount on the list price (markdown), and the higher the stock level the deeper the discount. Building on Federgruen and Heching's work, Chen and Simchi-Levi (2004a,b) include fixed ordering cost in the model. They derive the optimal pricing and replenishment strategies for both finite and infinite planning horizons. Feng and Chen (2003) study joint pricing and inventory optimization for a periodic review inventory system with the criterion of maximizing the long-run average profit. More recently, Allon and Zeevi (2011) addressed the simultaneous determination of pricing and capacity investment strategies for a multi-period inventory system and analyzed the optimal capacity, inventory, and pricing policies. For a detailed review on this stream of research, the reader is referred to Chen and Simchi-Levi (2010).

Different from the aforementioned two streams of research, our model integrates the two strategic decisions. We show that when the firm orders from both suppliers in period $n$, it should follow a base-stock type of policy with two thresholds $\left(s_{n}^{e}, s_{n}^{r}\right)$, that is, first raise the inventory level to $s_{n}^{e}$ by ordering from the expedited supply and then order from the regular supply to further raise the inventory position to $s_{n}^{r}$. And an optimal list price is set. If it is optimal for the firm to only order from the regular supply, the optimal policy is a state-dependent base-stock policy, with the base-stock level increasing in the starting inventory level. And a discount price is offered, which decreases with the starting inventory level. When the demand is modeled as an additive process, we show that the structure of the optimal policies can be further simplified. In particular, when only regular supply is used in the optimal policy, the base-stock level is increasing in the starting inventory level with slope between 0 and 1, and the order quantity is decreasing in the starting inventory level. Furthermore, we extend these results to the case with an infinite planning horizon. These results generalize those in Federgruen and Heching (1999).

We further study the operational impact of such supply diversification by considering the case where in the initial setting the firm has only one supplier, either the expedited supplier that has replenishment lead time 0 or the regular supplier that has lead time 1 , and analyzing the effect to the firm when it can source from both of them. We show that supply diversification increases the firm's profit, reduces the optimal safety-stock level (of the same supply source), and lowers the optimal selling price. Therefore, supply diversification benefits the firm as well as its customers. We conduct a numerical study to examine the respective benefit of supply diversification and dynamic pricing. We find that each strategy can increase the profitability of the firm quite significantly. In particular, supply diversification and dynamic pricing strategies seem to be "substitutes" for each other; in general, dual sourcing brings the firm more value than dynamic pricing unless the expedited supply is very costly. The intuition lies in the trade-off between a more flexible supply and the ability of managing demand; when the expedited ordering cost is very high, it will be rarely used. Hence, in that case, introducing the expedited supply mode adds little value to the system.

The remainder of this article is organized as follows. We first give a detailed description of the model and provide the formulation in the next section. In section 3, we present the main results on the optimal replenishment and pricing strategy for the finite horizon problem and then extend the results to an infinite horizon case. In section 4, we discuss the important special case of additive demand and present the simplified structure of the optimal inventory and pricing policies. In section 5, we analyze the effect of introducing a second supply and show that diversification is beneficial to both the firm and its customers. In section 6, we conduct a numerical study to demonstrate the results, and finally we conclude in section 7.

Throughout the article, we use "increasing" and "decreasing" in a non-strict sense, that is, they represent "nondecreasing" and "nonincreasing," respectively. 


\section{The Model}

Consider a firm managing a periodic-review inventory system with two supply modes: one is regular that has lead time 1, while the other is expedited that has lead time 0 . For convenience, we shall also call them regular and expedited/emergency suppliers. The planning horizon consists of $N$ periods, numbered in decreasing order, that is, the first period is $N$ and the last period is 1 . In each period $n$, the firm can order through regular supply at a unit ordering $\operatorname{cost} c_{n}^{r}$ or order through expedited supply at unit $\operatorname{cost} c_{n}^{e}$, where $c_{n}^{e}>c_{n}^{r}$. They are thereafter referred to as regular and expedited orders. Customer demand in period $n$ is random and sensitive to the selling price $p_{n}$. We use $D_{n}\left(p_{n}, \epsilon_{n}\right)$ to denote the random demand for each period $n$, where $\epsilon_{n}$ is a random noise and is independent from one another in different periods. $d_{n}\left(p_{n}\right)=\mathbf{E}\left[D_{n}\left(p_{n}, \epsilon_{n}\right)\right]$. Suppose $\underline{p} \leq p_{n} \leq \bar{p}$ for some given lower (upper limit) $\underline{p}(\bar{p})$. As in Federgruen and Heching (1999), we assume $D_{n}\left(p, \epsilon_{n}\right)$ is concave and decreasing in $p$ for any given sample path of $\epsilon_{n}$, so $d_{n}(p)$ is concave and decreasing in the selling price $p$. In each period, the firm determines the regular and expedited order quantities and sets the selling price for the product.

At the end of each period after demand is realized, the remaining inventory is carried over to the next period and incurs holding cost, while unsatisfied demand is backlogged and incurs shortage cost. Let $L_{n}(x)$ be the inventory holding and backlogging cost given the ending inventory level in period $n$ is $x$, and define

$$
G_{n}(y, p)=\mathbf{E}\left[L_{n}\left(y-D_{n}\left(p, \epsilon_{n}\right)\right)\right]
$$

where $y$ is the starting inventory level (after expedited ordering) at period $n$. Clearly, $G_{n}(y, p)$ represents the expected holding and shortage cost for period $n$ when the post-decision inventory level is $y$ and the selling price is $p$. Following Federgruen and Heching (1999), we assume that $G_{n}(y, p)$ is jointly convex in $y$ and $p$ and $\lim _{|y| \rightarrow \infty} G_{n}(y, p)=$ $\lim _{|y| \rightarrow \infty}\left[\left(c_{n}^{e}-c_{n}^{r}\right) y+G_{n}(y, p)\right]=\infty$ for any given $p \in[\underline{p}, \bar{p}]$.

The sequence of events is as follows. First, the firm receives the regular order placed in the previous period and updates and reviews the current inventory level; second, the expedited order is placed, if any, and then received; third, a regular order is placed and the selling price is set; fourth, demand is realized and excess demand is backlogged; fifth, all costs and revenue are incurred. The objective of the firm is to maximize the expected total discounted profit over a finite or an infinite horizon.

We summarize the additional notation that will be used subsequently as follows, in which the subscript $n$ is the index of period: $x_{n}=$ the initial inventory level before any decisions are made;

$y_{n}^{e}=$ the inventory level after placing the expedited order;

$y_{n}^{r}=$ the inventory position after placing the regular order;

$N=$ the length of the planning horizon;

$\alpha=$ the discount factor, $0 \leq \alpha<1$.

With the notation and the event sequence above, the order quantity from the expedited supply is $y_{n}^{e}-x_{n}$ while that from the regular supply is $y_{n}^{r}-y_{n}^{e}$.

Given the starting inventory level $x_{n}$, let $v_{n}\left(x_{n}\right)$ be the optimal expected discounted profit from period $n$ to 1 ; then the problem can be formulated as

$$
\begin{aligned}
v_{n}\left(x_{n}\right)= & \max _{y_{n}^{r} \geq y_{n}^{e} \geq x_{n}, p_{n} \in[p, \bar{p}]}\left\{p_{n} d\left(p_{n}\right)-c_{n}^{e}\left(y_{n}^{e}-x_{n}\right)\right. \\
& -c_{n}^{r}\left(y_{n}^{r}-y_{n}^{e}\right)-G_{n}\left(y_{n}^{e}, p_{n}\right) \\
& \left.+\alpha \mathbf{E}\left[v_{n-1}\left(y_{n}^{r}-D_{n}\left(p_{n}, \epsilon_{n}\right)\right)\right]\right\},
\end{aligned}
$$

and $v_{0}\left(x_{0}\right)=c_{0}^{e} x_{0}$, which means that at the end of the planning horizon, if $x_{0}>0$, the unit salvage value is $c_{0}^{e}$; otherwise, it costs $c_{0}^{e}$ to clear each unit of the backlog. The first term in Equation (1) is the expected revenue; the second and third terms are ordering cost from the expedited and regular supply, respectively; the fourth term is the expected inventory holding and demand backlogging cost; and the last term is the discounted expected profitto-go. Note that in each period $n$, as the regular order has lead time 1 , it has no impact on the inventory holding and backlogging cost in period $n$, whereas it affects the future periods by having the inventory level at the beginning of period $n-1$ at $y_{n}^{r}-D_{n}\left(p_{n}, \epsilon_{n}\right)$.

To facilitate the analysis, let $V_{n}\left(x_{n}\right)=v_{n}\left(x_{n}\right)-$ $c_{n}^{e} x_{n}, n \geq 0$, and so $V_{0}(x)=0$. From Equation (1), we have

$$
\begin{aligned}
v_{n}\left(x_{n}\right)-c_{n}^{e} x_{n}= & \max _{y_{n}^{r} \geq y_{n}^{e} \geq x_{n}, p_{n} \in[p, \bar{p}]}\left\{p_{n} d\left(p_{n}\right)-c_{n}^{e} y_{n}^{e}\right. \\
& -c_{n}^{r}\left(y_{n}^{r}-y_{n}^{e}\right)-G_{n}\left(y_{n}^{e}, p_{n}\right) \\
& +\alpha \mathbf{E}\left[v_{n-1}\left(y_{n}^{r}-D_{n}\left(p_{n}, \epsilon_{n}\right)\right)\right. \\
& \left.-c_{n-1}^{e}\left(y_{n}^{r}-D_{n}\left(p_{n}, \epsilon_{n}\right)\right)\right] \\
& \left.+\alpha c_{n-1}^{e} \mathbf{E}\left[\left(y_{n}^{r}-D_{n}\left(p_{n}, \epsilon_{n}\right)\right)\right]\right\} .
\end{aligned}
$$

From the definition of $V_{n}\left(x_{n}\right)$, we can get

$$
V_{n}\left(x_{n}\right)=\max _{y_{n}^{r} \geq y_{n}^{e} \geq x_{n}, p_{n} \in[\underline{p}, \bar{p}]}\left\{J_{n}\left(y_{n}^{e}, y_{n}^{r}, p_{n}\right)\right\},
$$


where for $n=2, \ldots, N$,

$$
\begin{aligned}
J_{n}\left(y^{e}, y^{r}, p\right)= & -\left(c_{n}^{e}-c_{n}^{r}\right) y^{e}+\left(\alpha c_{n-1}^{e}-c_{n}^{r}\right) y^{r} \\
& +\left(p-\alpha c_{n-1}^{e}\right) d(p)-G_{n}\left(y^{e}, p\right) \\
& +\alpha \mathbf{E}\left[V_{n-1}\left(y^{r}-D_{n}\left(p, \epsilon_{n}\right)\right)\right],
\end{aligned}
$$

and for $n=1$

$$
\begin{aligned}
J_{1}\left(y^{e}, y^{r}, p\right)= & -\left(c_{1}^{e}-c_{1}^{r}\right) y^{e}+\left(\alpha c_{0}^{e}-c_{1}^{r}\right) y^{r} \\
& +\left(p-\alpha c_{0}^{e}\right) d(p)-G_{1}\left(y^{e}, p\right) .
\end{aligned}
$$

This transformation essentially shifts the term $c_{n}^{e} x$ in Equation (1) to its previous period, period $n+1$, and will not alternate the structure of the optimal solutions. We can now consider $V_{n}\left(x_{n}\right)$ as the optimal value function and focus on $J_{n}\left(y^{e}, y^{r}, p\right)$ as the objective function for each period $n$.

Here we assume $\alpha c_{0}^{e} \leq c_{1}^{r}$ to prevent the firm from ordering an infinite amount from the regular supply in the last period. As a result, $y_{1}^{r}=y_{1}^{e}$ at optimum in period 1 as there is no reason to place a regular order. Furthermore, we assume $p \geq \alpha c_{n-1}^{e}$ for all $n$. In the rest of the article, for ease of exposition, we will omit the subscript $n$ unless confusion may arise.

\section{Optimal Operational Policy}

In this section, we characterize the optimal ordering and pricing strategies. In addition, we present some structural properties and quantitative relationships among the decisions.

First, we need the following technical result.

\section{LEMMA 1.}

(a) $J_{n}\left(y^{e}, y^{r}, p\right)$ is concave in $\left(y^{e}, y^{r}, p\right)$;

(b) $V_{n}(x)$ is concave and decreasing in $\mathrm{x}$.

ProOF. We prove the result by induction on $n$. Because $V_{0}(x)=0$, obviously (b) is true for $n=0$. For (a), in Equation (4), each term is concave, so (a) holds true for $n=1$. Suppose both (a) and (b) are valid for $n-1$. Because $G_{n}\left(y^{e}, p\right)$ is convex, $D_{n}(p, \epsilon)$ is concave in $p$, and $V_{n-1}(x)$ is decreasing concave (from the inductive assumption), $J_{n}\left(y^{e}, y^{r}, p\right)$ is concave in $\left(y^{e}, y^{r}, p\right)$. By Proposition-B4 in Heyman and Sobel (1984) that concavity is preserved after maximization, $V_{n}(x)$ is concave.

Finally, $V_{n}(x)$ is decreasing in $x$ because $J_{n}$ is independent of $x$ and a larger $x$ leads to a more restrictive feasible domain of $y^{r}$ and $y^{e}$ and so a smaller maximum objective function value.
For any given $y^{e}$ and $y^{r}$, define

$$
p_{n}\left(y^{e}, y^{r}\right)=\arg \max _{p \in[\underline{p}, \bar{p}]}\left\{J_{n}\left(y^{e}, y^{r}, p\right)\right\} .
$$

Substituting the optimal price $p_{n}\left(y^{e}, y^{r}\right)$ into Equation (3) yields

$$
\begin{aligned}
V_{n}(x)= & \max _{y^{r} \geq y^{e} \geq x}\left\{\left(c_{n}^{r}-c_{n}^{e}\right) y^{e}+\left(\alpha c_{n-1}^{e}-c_{n}^{r}\right) y^{r}\right. \\
& -G_{n}\left(y^{e}, p_{n}\left(y^{e}, y^{r}\right)\right)+\left(p_{n}\left(y^{e}, y^{r}\right)\right. \\
& \left.-\alpha c_{n-1}^{e}\right) d\left(p_{n}\left(y^{e}, y^{r}\right)\right) \\
& +\alpha \mathbf{E}\left[V_{n-1}\left(y^{r}-D_{n}\left(p_{n}\left(y^{e}, y^{r}\right), \epsilon_{n}\right)\right]\right\},
\end{aligned}
$$

which is concave in $\left(y^{e}, y^{r}\right)$ from Lemma 1(a). Define

$$
\begin{aligned}
\left(s_{n}^{e}, s_{n}^{r}\right) & =\arg \max _{y^{r} \geq y^{e}}\left\{J_{n}\left(y^{e}, y^{r}, p_{n}\left(y^{e}, y^{r}\right)\right)\right\} ; \\
S_{n}^{r}(x) & =\arg \max _{y^{r}}\left\{J_{n}\left(x, y^{r}, p_{n}\left(x, y^{r}\right)\right)\right\} .
\end{aligned}
$$

Let $\bar{p}_{n}=p_{n}\left(s_{n}^{e}, s_{n}^{r}\right)$ and $P_{n}(x)=p_{n}\left(x, S_{n}^{r}(x)\right)$. By their definitions, it is clear that $s_{n}^{r}=S_{n}^{r}\left(s_{n}^{e}\right)$ and $\bar{p}_{n}=P_{n}\left(s_{n}^{e}\right)$. With these, the optimal ordering and pricing strategy for each period is characterized in the following result.

THEOREM 1. The optimal ordering and pricing policy is characterized as follows. In each period $n$, if $x \leq s_{n}^{e}$, then $\left(y_{n}^{e^{*}}, y_{n}^{r^{*}}\right)=\left(s_{n}^{e}, s_{n}^{r}\right)$ and $p_{n}^{*}=\bar{p}_{n} ;$ if $x>s_{n}^{e}$, then if $x<S_{n}^{r}(x),\left(y_{n}^{e^{*}}, y_{n}^{r^{*}}\right)=\left(x, S_{n}^{r}(x)\right)$ and $p_{n}^{*}=P_{n}(x)$; otherwise, $\left(y_{n}^{e^{*}}, y_{n}^{r^{*}}\right)=(x, x)$ and $p_{n}^{*}=p_{n}(x, x)$.

Proof. The result follows from the concavity of $J_{n}\left(y^{e}, y^{r}, p\right)$ and definitions (5)-(7).

The theorem shows that, when $x \leq s_{n}^{e}$, the optimal inventory replenishment policy is a state-independent base-stock type with two thresholds, one for expedited order and the other for regular order; when $x>s_{n}^{e}$, the firm should only order from the regular supply using a state-dependent base-stock policy. Specifically, if the starting inventory level $x$ at the beginning of period $n$ is less than $s_{n}^{e}$, it is optimal to order up to $s_{n}^{e}$ using the expedited order, then use the regular order to raise the inventory position to $s_{n}^{r}$, and to sell the product at price $\bar{p}_{n}$; if the starting inventory level $x$ is greater than $s_{n}^{e}$, then only use the regular order to bring the inventory position to $\max \left\{x, S_{n}^{r}(x)\right\}$ and set the price at $p_{n}\left(x, \max \left\{x, S_{n}^{r}(x)\right\}\right)$.

When the value and cost functions are continuous and differentiable, due to the concavity of $J_{n}\left(y^{e}, y^{r}, p_{n}\left(y^{e}, y^{r}\right)\right),\left(s_{n}^{e}, s_{n}^{r}\right)$ can be solved by the corresponding first order conditions. Specifically, when $n=1, s_{n}^{e}=s_{n}^{r}$ and satisfies 


$$
-\left(c_{n}^{e}-\alpha c_{n-1}^{e}\right)-\left(G_{n}\left(y, p_{n}(y, y)\right)_{y=s_{n}^{e}}^{\prime}=0\right.
$$

which has a finite solution because of $c_{1}^{e}>c_{1}^{r} \geq \alpha c_{0}^{e}$ and the assumption on $G_{n}(y, p)$; when $n>1$, denote $\left(\bar{y}^{e}, \bar{y}^{r}\right)$ the solutions to the following set of equations:

$$
\begin{gathered}
-\left(c_{n}^{e}-c_{n}^{r}\right)-\left(G_{n}\left(y^{e}, p_{n}\left(y^{e}, y^{r}\right)\right)_{y^{e}}^{\prime}=0\right. \\
\alpha c_{n-1}^{e}-c_{n}^{r}+\left(\alpha \mathbf{E}\left[V_{n-1}\left(y^{r}-D\left(p_{n}\left(y^{e}, y^{r}\right), \epsilon\right)\right)\right]\right)_{y^{r}}^{\prime}=0
\end{gathered}
$$

If $\bar{y}^{e} \leq \bar{y}^{r}$, then $s_{n}^{e}=\bar{y}^{e}$ and $s_{n}^{r}=\bar{y}^{r}$; otherwise $s_{n}^{e}=s_{n}^{r}$ and it satisfies

$$
\begin{aligned}
& -\left(c_{n}^{e}-\alpha c_{n-1}^{e}\right)+\left(-G_{n}\left(y, p_{n}(y, y)\right)\right. \\
& \left.\quad+\alpha \mathbf{E}\left[V_{n-1}\left(y-D\left(p_{n}(y, y), \epsilon\right)\right)\right]\right)_{y=s_{n}^{e}}^{\prime}=0 .
\end{aligned}
$$

It can be seen from Equation (10) that if $\alpha c_{n-1}^{e} \leq c_{n}^{r}$, then $\bar{y}^{e^{*}}>\bar{y}^{r^{*}}$ and so $s_{n}^{r}=s_{n}^{e}$ because the left hand side of Equation (10) would be negative in $y^{r}$.

And $S_{n}^{r}(x)$ is the solution of

$$
\alpha c_{n-1}^{e}-c_{n}^{r}+\left(\alpha \mathbf{E}\left[V_{n-1}\left(y-D\left(p_{n}(x, y), \epsilon\right)\right)\right]\right)_{y}^{\prime}=0 .
$$

After solving $s_{n}^{e}, s_{n}^{r}$, and $S_{n}^{r}(x)$, we can find the optimal price $\bar{p}_{n}$ and $p_{n}\left(y^{e}, y^{r}\right)$.

\section{LEMMA 2.}

(a) $J_{n}\left(y^{e}, y^{r}, p\right)$ is a submodular function in $y^{e}$ and $p$.

(b) $J_{n}\left(y^{e}, y^{r}, p\right)$ is a submodular function in $y^{r}$ and $p$.

(c) $J_{n}\left(y^{e}, y^{r}, p_{n}\left(y^{e}, y^{r}\right)\right)$ is a supermodular function in $y^{e}$ and $y^{r}$.

\section{ProOF.}

(a) For $n=1$, because each of the first three terms in $J_{1}\left(y^{e}, y^{r}, p\right)$ either depends only on $y^{e}$ or $p$ or is a constant with respect to $y^{e}$ and $p$, it suffices to show that $-G_{1}(y, p)$ is submodular or $G_{1}(y, p)$ is supermodular. Note that $G_{n}(y, p)=$ $\mathbf{E}\left[L_{n}\left(y-D_{n}(p, \epsilon)\right)\right]$ where $L_{n}(\cdot)$ is convex and $D_{n}(p, \epsilon)$ is decreasing in $p$. Therefore, $L_{n}(y-D(p, \epsilon))$ is supermodular in $(y, p)$ (see e.g., Theorem 2 in Federgruen and Heching 1999) and so is $G_{n}(y, p)$. For $n>1$, as the only term that depends on both $y^{e}$ and $p$ is $G_{n}(y, p)$, the same argument above leads to the submodularity of $J_{n}\left(y^{e}, y^{r}, p\right)$. So part (a) follows.

(b) For $n=1$, the result is obviously true. For $n>1$, we just need to show the submodularity of $V_{n}\left(y-D_{n}(p, \epsilon)\right)$ in $(y, p)$. Again, by the concavity of $V_{n}(x)$ and the monotonicity of $D_{n}(p, \epsilon)$, the result follows. (c) First note that the terms related to $y^{e}$ or $y^{r}$ are separable in $J_{n}\left(y^{e}, y^{r}, p\right)$ and so $J_{n}\left(y^{e}, y^{r}, p\right)$ is supermodular in $\left(y^{e}, y^{r}\right)$. Let $\tilde{p}=-p$ and define $\tilde{J}_{n}\left(y^{e}, y^{r}, \tilde{p}\right)=J_{n}\left(y^{e}, y^{r},-\tilde{p}\right)$. From parts (a) and (b), $\tilde{J}_{n}\left(y^{e}, y^{r}, \tilde{p}\right)$ is supermodular in $\left(y^{e}, \tilde{p}\right)$ and $\left(y^{r}, \tilde{p}\right)$. Therefore, $\tilde{J}_{n}\left(y^{e}, y^{r}, \tilde{p}\left(y^{e}, y^{r}\right)\right)=$ $\max _{\tilde{p} \in[-\bar{p},-\underline{p}]} \tilde{J}_{n}\left(y^{e}, y^{r}, \tilde{p}\right)$ is supermodular in $\left(y^{e}, y^{r}\right)$ as the constraint set of $\tilde{p}$ is a lattice (see Theorem 2.7.6 in Topkis 1998). Therefore, $J_{n}\left(y^{e}, y^{r}, p\left(y^{e}, y^{r}\right)\right)$ is supermodular.

The following property of the optimal policy parameters is a direct consequence of Lemma 2 (see Topkis 1998).

\section{THEOREM 2.}

(a) Given $y_{n}^{e}, p_{n}\left(y_{n}^{e}, y_{n}^{r}\right)$ is decreasing in $y_{n}^{r}$.

(b) Given $y_{n}^{r}, p_{n}\left(y_{n}^{e}, y_{n}^{r}\right)$ is decreasing in $y_{n}^{e}$.

(c) $S_{n}^{r}(x)$ is increasing in $x$ and $P_{n}(x)$ is decreasing in $x$.

The trajectory of the optimal price with respect to the starting inventory level can be summarized as follows. When $x \leq s_{n}^{e}$, the optimal price is a constant at $\bar{p}_{n}$; when $s_{n}^{e}<x \leq S_{n}^{r}(x), p_{n}^{*}=P_{n}(x)$; when $x>S_{n}^{r}(x)$, $p_{n}^{*}=p_{n}(x, x)$. The theorem shows that the optimal selling price is decreasing in the starting inventory level $x$. More important, from Theorem 2 and the definitions of $\bar{p}_{n}, P_{n}(x)$, and $p_{n}(x, x)$, the firm would charge a lower price (discount) when the starting inventory level increases. By considering $\bar{p}_{n}$ as the list price, the optimal pricing strategy for the firm is still a list-price policy. This extends the result of Federgruen and Heching (1999) to a model with two supply modes.

In what follows we extend the results to the case with an infinite planning horizon. All the cost parameters and demand distribution are stationary. In the analysis of infinite horizon models it is easier to have the one-period reward uniformly non-positive so that the results in negative dynamic programming can be applied. Since the original problem has no such property, we subtract a constant

$$
M=\max _{\underline{p} \leq p \leq \bar{p}} p d(p),
$$

which is assumed to be finite, from the original oneperiod expected profit (for period $n \geq 1$ ). We then obtain the transformed value function $\bar{V}_{n}(x)$ for the finite horizon problem from the original value function $V_{n}(x)$ :

$$
\bar{V}_{n}(x)=V_{n}(x)-\frac{M\left(1-\alpha^{n}\right)}{1-\alpha}
$$


and

$$
\bar{J}_{n}\left(y^{e}, y^{r}, p\right)=J_{n}\left(y^{e}, y^{r}, p\right)-\frac{M\left(1-\alpha^{n}\right)}{1-\alpha} .
$$

Consequently, for the transformed model, each period's reward is non-positive and $\bar{V}_{0}(x)=0$. So the optimal value function of the infinite horizon problem $\bar{V}(x)$ satisfies the optimality equations (e.g., Proposition 3.1.1, Bertsekas 2007):

$$
\bar{V}(x)=\max _{y^{r} \geq y^{e} \geq x, p \in[\underline{p}, \bar{p}]} \bar{J}\left(y^{e}, y^{r}, p\right)
$$

where

$$
\begin{aligned}
\bar{J}\left(y^{e}, y^{r}, p\right)= & \left(c^{r}-c^{e}\right) y^{e}+\left(\alpha c^{e}-c^{r}\right) y^{r}+\left(p-\alpha c^{e}\right) d(p) \\
& -G\left(y^{e}, p\right)-M+\alpha \mathbf{E}\left[\bar{V}\left(y^{r}-D(p, \epsilon)\right)\right] .
\end{aligned}
$$

In the following, we first present the relationship between $\bar{V}$ and $\bar{J}$ and $\bar{V}_{n}$ and $\bar{J}_{n}$ as well as those of the original problem.

\section{PROPOSITION 1.}

(a) $\bar{V}(x)=\lim _{n \rightarrow \infty} \bar{V}_{n}(x), \quad V(x)=\lim _{n \rightarrow \infty} V_{n}(x)$, $\bar{J}\left(y^{e}, y^{r}, p\right)=\lim _{n \rightarrow \infty} \bar{J}_{n}\left(y^{e}, y^{r}, p\right)$,

$J\left(y^{e}, y^{r}, p\right)=\lim _{n \rightarrow \infty} J_{n}\left(y^{e}, y^{r}, p\right) ;$ and

$\bar{V}(x)=V(x)-\frac{M}{1-\alpha}$ and

$\bar{J}\left(y^{e}, y^{r}, p\right)=J\left(y^{e}, y^{r}, p\right)-\frac{M}{1-\alpha}$.

(b) $V$ and $J$ satisfy the optimality equation

$$
V(x)=\max _{y^{r} \geq y^{e} \geq x, p \in[\underline{p}, \bar{p}]}\left\{J\left(y^{e}, y^{r}, p\right)\right\}
$$

where

$$
\begin{aligned}
J\left(y^{e}, y^{r}, p\right)= & \left(c^{r}-c^{e}\right) y^{e}+\left(\alpha c^{e}-c^{r}\right) y^{r}+\left(p-\alpha c^{e}\right) d(p) \\
& -G\left(y^{e}, p\right)+\alpha \mathbf{E}\left[V\left(y^{r}-D(p, \epsilon)\right)\right] .
\end{aligned}
$$

(c) $V(x)$ is concave and decreasing in $x$ and $J\left(y^{e}, y^{r}, p\right)$ is concave in $\left(y^{e}, y^{r}, p\right)$.

Proof. From Lemma 1, $\bar{J}_{n}\left(y^{e}, y^{r}, p\right)$ is concave and so is continuous. The assumption on $G(y, p)$ implies that, for each $p \in[p, \bar{p}], \bar{J}_{n}\left(y^{e}, y^{r}, p\right) \rightarrow \infty$ when $\left\|\left(y^{e}, y^{r}\right)\right\| \rightarrow \infty$ (here $\|\cdot\|^{-}$is Euclidean norm). Because the transformed problem belongs to negative dynamic program, based on Proposition 3.1.7 in Bertsekas (2007) that value iteration converges to the optimal value function $\bar{V}(x)$, parts (a) and (b) follow. For part (c), $V(x)$ and $J\left(y^{e}, y^{r}, p\right)$ just inherit the properties of $V_{n}(x)$ and $J_{n}\left(y^{e}, y^{r}, p\right)$.
Define

$$
\begin{aligned}
p\left(y^{e}, y^{r}\right) & =\arg \max _{p \in[p, \bar{p}]}\left\{J\left(y^{e}, y^{r}, p\right)\right\}, \\
\left(s^{e}, s^{r}\right) & =\arg \max _{y^{r} \geq y^{e}}\left\{J\left(y^{e}, y^{r}, p\left(y^{e}, y^{r}\right)\right)\right\}, \\
S^{r}(x) & =\arg \max _{y^{r}}\left\{J\left(x, y^{r}, p\left(x, y^{r}\right)\right)\right\} .
\end{aligned}
$$

Similarly, we can obtain the following results from Lemma 2 in the finite horizon case and the value iteration.

\section{PROPOSITION 2.}

(a) $J\left(y^{e}, y^{r}, p\right)$ is submodular in $\left(y^{e}, p\right)$ and $\left(y^{r}, p\right)$;

(b) $J\left(y^{e}, y^{r}, p\left(y^{e}, y^{r}\right)\right)$ is supermodular in $\left(y^{e}, y^{r}\right)$.

The next result characterizes the optimal policy.

THEOREM 3. There exists a stationary optimal base-stock and list-price policy for the infinite horizon model, which can be characterized as

$$
\begin{aligned}
& \left(y^{e^{*}}, y^{r^{*}}, p^{*}\right) \\
& \quad= \begin{cases}\left(s^{e}, s^{r}, \bar{p}\right) & \text { if } x \leq s^{e} \\
\left(x, S^{r}(x), p\left(x, S^{r}(x)\right)\right) & \text { if } S^{r}(x) \geq x>s^{e} \\
(x, x, p(x, x)) & \text { if } x>S^{r}(x) .\end{cases}
\end{aligned}
$$

Proof. From Propositions 3.1.3 and 3.1.7 of Bertsekas (2007), there exists a stationary optimal policy for such a negative dynamic program, and the policy maximizes the right-hand side of the optimality equation (12). So the result follows.

\section{Additive Demand}

In this section, we consider a special case of the model with additive demand, that is, the demand function $D_{n}\left(p, \epsilon_{n}\right)$ is separable and can be written as

$$
D_{n}\left(p, \epsilon_{n}\right)=d_{n}(p)+\epsilon_{n},
$$

where $d_{n}(p)$ is decreasing in $p$ and $\mathbf{E}\left[\epsilon_{n}\right]=0$. Additive demand implies that the probability of $D_{n}\left(p, \epsilon_{n}\right)$ differing from $d_{n}(p)$ by any amount is independent of the price $p$ (Mills 1959), and it has been widely used and discussed in the literature on joint inventory and pricing optimization (e.g., Chen and Simchi-Levi 2004a,b). Let $g_{n}(d)$ denote the inverse function of $d_{n}(p)$, which is well defined as $d_{n}(p)$ is a decreasing function of $p$. As in Chen and SimchiLevi (2004a), we assume that the expected revenue function $R_{n}(d)=d g_{n}(d)$ is concave in $d$. Under the additive demand function, we are able to provide a more detailed characterization of the optimal policy. 
Because when $p$ increases from $\underline{p}$ to $\bar{p}, d_{n}(p)$ decreases from $\bar{d}_{n}=d_{n}(p)$ to $\underline{d}_{n}=d_{n}(\bar{p})$, the decision variable can be changed from $p$ to $d$, and the optimality equation can now be written as

$$
\begin{aligned}
v_{n}(x)= & \max _{y^{r} \geq y^{e} \geq x, d \in\left[\underline{d}_{n}, \bar{d}_{n}\right]}\left\{R_{n}(d)-c_{n}^{e}\left(y^{e}-x\right)-c_{n}^{r}\left(y^{r}-y^{e}\right)\right. \\
& \left.-G_{n}\left(y^{e}-d\right)+\alpha \mathbf{E}\left[v_{n-1}\left(y^{r}-d-\epsilon_{n}\right)\right]\right\},
\end{aligned}
$$

where

$$
G_{n}(y)=\mathbf{E}\left[L_{n}\left(y-\epsilon_{n}\right)\right]
$$

Through observing Equation (13), we conduct another change of variable $\tilde{y}^{e}=y^{e}-d$ and $\tilde{y}^{r}=y^{r}-d$; then the optimality equation (13) can be expressed as

$$
\begin{aligned}
v_{n}(x)= & \max _{\tilde{y}^{r} \geq \tilde{y}^{e} \geq x-d, d \in\left[\underline{d}_{n}, \bar{d}_{n}\right]}\left\{\left[R_{n}(d)-c_{n}^{e} d\right]-\left(c_{n}^{e}-c_{n}^{r}\right) \tilde{y}^{e}\right. \\
& \left.-G_{n}\left(\tilde{y}^{e}\right)-c_{n}^{r} \tilde{y}^{r}+\alpha \mathbf{E}\left[v_{n-1}\left(\tilde{y}^{r}-\epsilon_{n}\right)\right]\right\}+c_{n}^{e} x .
\end{aligned}
$$

As seen in the previous section, all the value functions involved can be inductively shown to be concave. Define $H_{n}\left(\tilde{y}^{r}\right)=-c_{n}^{r} \tilde{y}^{r}+\alpha \mathbf{E}\left[v_{n-1}\left(\tilde{y}^{r}-\epsilon_{n}\right)\right]$, and let $\tilde{y}_{n}^{2}$ be its maximizer. Then $\max _{\tilde{y}^{r} \geq \tilde{y}^{e}} H_{n}\left(\tilde{y}^{r}\right)=$ $H_{n}\left(\tilde{y}^{e} \vee \tilde{y}_{n}^{2}\right)$, where " $\vee$ " is the maximum operator, that is, for any real numbers $x$ and $y, x \vee y=\max \{x, y\}$. Thus we can further rewrite the optimality equation as

$$
\begin{aligned}
v_{n}(x)= & \max _{\tilde{y}^{e} \geq x-d, d \in\left[\underline{d}_{n}, \bar{d}_{n}\right]}\left\{\left[R_{n}(d)-c_{n}^{e} d\right]-\left(c_{n}^{e}-c_{n}^{r}\right) \tilde{y}^{e}\right. \\
& \left.-G_{n}\left(\tilde{y}^{e}\right)+\max _{\tilde{y}^{r} \geq \tilde{y}^{e}}\left\{-c_{n}^{r} \tilde{y}^{r}+\alpha \mathbf{E}\left[v_{n-1}\left(\tilde{y}^{r}-\epsilon_{n}\right)\right]\right\}\right\} \\
& +c_{n}^{e} x \\
= & \max _{\tilde{y}^{e} \geq x-d, d \in\left[\underline{d}_{n}, \bar{d}_{n}\right]}\left\{\left[R_{n}(d)-c_{n}^{e} d\right]-\left(c_{n}^{e}-c_{n}^{r}\right) \tilde{y}^{e}\right. \\
& \left.-G_{n}\left(\tilde{y}^{e}\right)+H_{n}\left(\tilde{y}^{e} \vee \tilde{y}_{n}^{2}\right)\right\}+c_{n}^{e} x .
\end{aligned}
$$

Let $\tilde{y}_{n}^{1}$ be the maximizer of

$$
W_{n}\left(\tilde{y}^{e}\right)=-\left(c_{n}^{e}-c_{n}^{r}\right) \tilde{y}^{e}-G_{n}\left(\tilde{y}^{e}\right)+H_{n}\left(\tilde{y}^{e} \vee \tilde{y}_{n}^{2}\right) .
$$

Then

$$
\max _{\tilde{y}^{e} \geq x-d}\left\{W_{n}\left(\tilde{y}^{e}\right)\right\}=W_{n}\left((x-d) \vee \tilde{y}_{n}^{1}\right) .
$$

Consequently, the optimal expected demand $d$ (or the optimal price $p=g_{n}(d)$ ) can be identified by solving the following problem:

$$
v_{n}(x)=\max _{d \in\left[d_{n}, \tilde{d}_{n}\right]}\left\{\left[R_{n}(d)-c_{n}^{e} d\right]+W_{n}\left((x-d) \vee \tilde{y}_{n}^{1}\right)\right\}
$$

Define

$$
\tilde{d}_{n}(x)=\arg \max _{d \in\left[\mathbb{d}_{n}, \tilde{d}_{n}\right]}\left\{\left[R_{n}(d)-c_{n}^{e} d\right]+W_{n}\left((x-d) \vee \tilde{y}_{n}^{1}\right)\right\} .
$$

LEMMA 3. $\tilde{d}_{n}(x)$ is increasing in $x$ with slope no more than 1.

Proof. The objective function on the right-hand side of Equation (16) is a concave function of $d$, and it is supermodular in $(x, d)$, and the constraint $d \in\left[\underline{d}_{n}, \bar{d}_{n}\right]$ is a lattice (see Topkis 1998 or Porteus 2002). Thus the optimal solution $\tilde{d}_{n}(x)$ is an increasing function of $x$. Letting $z=x-d$, then the optimization problem (16) can be written as

$$
\begin{aligned}
v_{n}(x)= & \max _{x-\bar{d}_{n} \leq z \leq x-\underline{d}_{n}}\left\{\left[R_{n}(x-z)-c_{n}^{e}(x-z)\right]\right. \\
& \left.+W_{n}\left(z \vee \tilde{y}_{n}^{1}\right)\right\},
\end{aligned}
$$

where the bracketed terms are supermodular in $(x, z)$. Because the constraint $\left\{(x, z) \mid x-\bar{d}_{n} \leq z \leq x-\underline{d}_{n}\right\}$ is a lattice, it follows from Lemma 8.4 of Porteus (2002) that the optimal solution $z_{n}^{*}(x)=x-\tilde{d}_{n}(x)$ is increasing in $x$. This shows $0 \leq \tilde{d}_{n}(x+1)-\tilde{d}_{n}(x) \leq 1$.

The one-period expected profit function $R_{n}(d)-c_{n}^{e} d$ is concave, and we define

$$
d_{n}^{*}=\arg \max _{d \in\left[\mathbb{d}_{n}, \bar{d}_{n}\right]}\left\{R_{n}(d)-c_{n}^{e} d\right\} .
$$

Thus, if $x \leq \tilde{y}_{n}^{1}+d_{n}^{*}$, then the optimal pricing decision in Equation (16) is $d_{n}^{*}$, while if $x>\tilde{y}_{\mathfrak{u}}^{1}+d_{n}^{*}$, then the optimal solution is $\tilde{d}_{n}(x)$ satisfies $x-\tilde{d}_{n}(x) \geq \tilde{y}_{n}^{1}$.

Based on the preceding analysis, we characterize the optimal policies in the next theorem. Let $p_{n}^{*}=g_{n}\left(d_{n}^{*}\right)$ and $p_{n}(x)=g_{n}\left(\tilde{d}_{n}(x)\right)$ for $x>y_{n}^{1}+d_{n}^{*}$.

THeOREM 4. When demand is additive, the optimal inventory control policy for period $n$ is determined by two critical numbers $y_{n}^{1} \leq y_{n}^{2}$, and the optimal pricing policy is a list price with markdown policy, with a list price $p_{n}^{*}$ and markdowns determined by a decreasing function $p_{n}(x) \leq p_{n}^{*}$ for $x>y_{n}^{1}+d_{n}^{*}$. More specifically, suppose the starting inventory level at period $n$ is $x$;

(i) if $x \leq y_{n}^{1}+d_{n}^{*}$, then the optimal inventory policy is determined by two base-stock levels $\left(y_{n}^{e^{*}}, y_{n}^{r^{*}}\right)=$ $\left(y_{n}^{1}+d_{n}^{*}, y_{n}^{2}+d_{n}^{*}\right)$ such that the firm orders through the expedited supply to raise the inventory level to the base-stock level $y_{n}^{1}+d_{n}^{*}$, then orders through the 
regular supply to raise the inventory position to the base-stock level $y_{n}^{2}+d_{n}^{*}$ (i.e., order $y_{n}^{2}-y_{n}^{1}$ from the regular supply), and the optimal price is $p_{n}^{*}$;

(ii) if $y_{n}^{1}+d_{n}^{*}<x$, then only order through the regular supply to raise the inventory position to $\max \left\{x, y_{n}^{2}+\tilde{d}_{n}(x)\right\}$, viz., $\left(y_{n}^{e^{*}}, y_{n}^{r^{*}}\right)=\left(x, \max \left\{x, y_{n}^{2}+\right.\right.$ $\left.\left.\tilde{d}_{n}(x)\right\}\right)$ and set a markdown price $p_{n}(x)$; the basestock level $y_{n}^{2}+\tilde{d}_{n}(x)$ is increasing in the starting inventory level $x$ at slope no more than 1 , and the optimal order quantity from the regular supply, $y_{n}^{2}+$ $\tilde{d}_{n}(x)-x$, is decreasing in $x$ with slope at least -1 .

PROOF. Let $y_{n}^{1}=\tilde{y}_{n}^{1}$ and $y_{n}^{2}=\max \left\{\tilde{y}_{n}^{2}, y_{n}^{1}\right\}$; thus $y_{n}^{2} \geq y_{n}^{1}$.

Case 1. $y_{n}^{1} \leq \tilde{y}_{n}^{2}$. In this case, $y_{n}^{2}=\tilde{y}_{n}^{2}$. If the starting inventory level $x_{n} \leq y_{n}^{1}+d_{n}^{*}$, then as we discussed previously, the optimal pricing decision is $p_{n}^{*}\left(d_{n}^{*}\right)$ and, by Equation (16), the optimal $\tilde{y}_{n}^{e}$ in Equation (15) is $y_{n}^{1}$. Thus the optimal expedited order decision is $y_{n}^{e^{*}}=y_{n}^{1}+d_{n}^{*}$. This shows that the optimal ordering decision is to raise the inventory level to $y_{n}^{1}+d_{n}^{*}$ by the expedited supply. Next, by Equation (14), the optimal $\tilde{y}_{n}^{r}$ is $y_{n}^{2}$; thus the optimal $y_{n}^{r^{*}}=y_{n}^{2}+d_{n}^{*}$. This implies that it is optimal to raise the inventory position to $y_{n}^{2}+d_{n}^{*}$ by ordering $y_{n}^{2}-y_{n}^{1}$ units from the regular supply.

If $x_{n}>y_{n}^{1}+d_{n}^{*}$ but $x_{n} \leq y_{n}^{2}+\tilde{d}_{n}\left(x_{n}\right)$, then by Equation (15), the optimal $\tilde{y}_{n}^{e}$ is $x_{n}-\tilde{d}_{n}\left(x_{n}\right)$, or the optimal $y_{n}^{e}$ is $x_{n}$, meaning that the firm does not resort to expedited supply. The optimal price in this case is $p_{n}(x)$ (the average demand is $\tilde{d}_{n}\left(x_{n}\right)$ ). By substituting this into Equation (14), it is seen that the optimal $\tilde{y}_{n}^{r}$ is $y_{n}^{2}$; thus the optimal $y_{n}^{r^{*}}=y_{n}^{2}+\tilde{d}_{n}\left(x_{n}\right)$, which is an increasing function of $x_{n}$ but with slope no more than 1 . Thus in this case, the optimal decision is to only use the regular supply to raise the inventory position to $y_{n}^{2}+\tilde{d}_{n}\left(x_{n}\right)$ (by ordering $\left.y_{n}^{2}+\tilde{d}_{n}\left(x_{n}\right)-x_{n}\right)$, and to not resort to expedited supply. Since the $\tilde{d}_{n}\left(x_{n}\right)$ is increasing with slope no more than 1 , it is clear that the order quantity $y_{n}^{2}+\tilde{d}_{n}\left(x_{n}\right)-x_{n}$ is decreasing with slope at least -1 .

Finally, if $x_{n}>y_{n}^{2}+\tilde{d}_{n}\left(x_{n}\right)$, then it is seen from Equations (14) and (15) that the optimal $\tilde{y}_{n}^{e}$ and $\tilde{y}_{n}^{r}$ are both equal to $x_{n}-\tilde{d}_{n}\left(x_{n}\right)$; thus $\left(y_{n}^{e^{*}}, y_{n}^{r^{*}}\right)=(x, x)$. This shows that the firm does not order anything.

Case 2. $y_{n}^{1}>\tilde{y}_{n}^{2}$. Then $y_{n}^{2}=y_{n}^{1}$. From the definition of $y_{n}^{1}$, it implies that $\tilde{y}^{e}=\tilde{y}^{r}$ or the firm does not need to resort to the regular supply. Therefore, if the starting inventory level $x_{n} \leq y_{n}^{1}+d_{n}^{*}$, then the optimal replenishment level through expedited order is $y_{n}^{1}+d_{n}^{*}$, while if $x_{n}>y_{n}^{1}+d_{n}^{*}$ (and so $y_{n}^{1}+d_{n}^{*} \geq y_{n}^{1}$ $+\tilde{d}_{n}\left(x_{n}\right)$ ), then by Equations (14) and (15), no order is placed. This policy is exactly the same as what would be achieved using the policy as described in the theo- rem, but using optimal control parameters $y_{n}^{1}=y_{n}^{2}$. The proof is now complete.

Note that if $y_{n}^{1}=y_{n}^{2}$, then regular supply mode will not be resorted to in period $n$ regardless of the starting inventory level. And compared with the results in section 3, we can see that $s_{n}^{e}=y_{n}^{1}+d_{n^{\prime}}^{*} s_{n}^{r}=y_{n}^{2}+d_{n^{\prime}}^{*}$ and $S_{n}^{r}(x)=y_{n}^{2}+\tilde{d}_{n}(x)$. Furthermore, the computation of the control parameters is easier in this additive demand case because they can be solved sequentially through several single-dimension concave optimization problems.

REMARK 1 . The optimal pricing policy in Theorem 4 is list price with markdowns. It should be noted, however, that the list-price structure remains only when the expedited supply mode exists. When expedited supply mode is removed, the optimal pricing policy will no longer be of a list-price type. See the next section for more discussion on this.

REMARK 2. In the special case of $\underline{d}_{n}=\bar{d}_{n}$, the average demand and the selling price are fixed, and our model, as well as results, reduces to that of Fukuda (1964). Therefore, Theorem 4 generalizes Fukuda's result to the joint optimization of inventory and pricing. The optimal inventory decisions in Theorem 4 remains the same as that in Fukuda (1964), and it is a dual base-stock policy.

REMARK 3. The two critical numbers $y_{n}^{1}$ and $y_{n}^{2}$ can be interpreted as the safety-stock level and safetystock position, respectively, for period $n$. To see this, note that at the beginning of period $n$, if the firm orders from the expedited supply, it raises the inventory level to $y_{n}^{1}+d_{n}^{*}$ and then orders from the regular supply to raise the inventory position to $y_{n}^{2}+d_{n}^{*}$. As $d_{n}^{*}$ is the average demand for period $n$, the expected inventory level at the end of period $n$ is $y_{n}^{1}$, which is the safety-stock level. Similarly, $y_{n}^{2}$ is the average inventory position at the end of period $n$ (when only the regular supply is used, $y_{n}^{2}$ is still the average inventory position at the end of period $n$ as $\tilde{d}_{n}(x)$ is the average demand), and we call it the safety-stock position for period $n$.

\section{Operational Impact of Supply Diversification}

Most firms in practice have more than one supply source. One interesting question to ask is what the effect is to the firm when an additional supply source is introduced. In this section we address this issue. As in the previous section, the demand function is assumed to be additive. 


\subsection{Impact of Adding the Regular Supply}

We first consider the case where, initially, the firm only has the expedited supply with lead time 0 , and we want to study the impact to the firm after introducing the regular supply that has replenishment lead time 1.

When the firm has only the expedited supply, we use $\hat{v}_{n}(x)$ to represent its maximum expected total discounted profit function. The corresponding optimality equation is

$$
\begin{aligned}
\hat{v}_{n}(x)= & \max _{y^{e} \geq x-d, d \in\left[d_{n}, \bar{d}_{n}\right]}\left\{\left[R_{n}(d)-c_{n}^{e} d\right]-c_{n}^{e} y^{e}-G_{n}\left(y^{e}\right)\right. \\
& \left.+\alpha \mathbf{E}\left[\hat{v}_{n-1}\left(y^{e}-\epsilon_{n}\right)\right]\right\}+c_{n}^{e} x .
\end{aligned}
$$

The boundary condition is $\hat{v}_{0}(x)=c_{0}^{e} x$.

The optimal policy for this case can be obtained as a special case of the analysis in the previous section. Specifically, if the starting inventory level in period $n$ is less than $\hat{y}_{n}^{1}+d_{n}^{*}$ (note that $d_{n}^{*}$ is defined as in Equation (17)) where

$$
\hat{y}_{n}^{1}=\arg \max _{y^{e}}\left\{-c_{n}^{e} y^{e}-G_{n}\left(y^{e}\right)+\alpha \mathbf{E}\left[\hat{v}_{n-1}\left(y^{e}-\epsilon_{n}\right)\right]\right\},
$$

then the firm raises the inventory level to $\hat{y}_{n}^{1}+d_{n^{\prime}}^{*}$ and the optimal selling price is the list price $\hat{p}_{n}^{*}=g_{n}\left(d_{n}^{*}\right)$; if the starting inventory level is over $\hat{y}_{n}^{1}+d_{n}^{*}$, then the firm orders nothing and the optimal selling price is $\hat{p}_{n}(x) \leq \hat{p}_{n}^{*}$ where $\hat{p}_{n}(x)=$ $g_{n}\left(\hat{d}_{n}(x)\right)$ and

$$
\begin{aligned}
\hat{d}_{n}(x)= & \arg \max _{d \in\left[\underline{d}_{n}, \hat{d}_{n}\right]}\left\{\left[R_{n}(d)-c_{n}^{e} d\right]-c_{n}^{e}(x-d)\right. \\
& \left.-G_{n}(x-d)+\alpha \mathbf{E}\left[\hat{v}_{n-1}\left(x-d-\epsilon_{n}\right)\right]\right\}
\end{aligned}
$$

and $\hat{p}_{n}(x)$ is decreasing in $x$.

We shall use the same notation for, for example, value function, optimal policies, etc., as in the previous section for the system with both the expedited and regular supply modes. Recall that the optimal value function for the system with dual supply modes is $v_{n}(x)$ and its optimal policy in period $n$ is determined by $y_{n}^{1} \leq y_{n}^{2}, d_{n}^{*}\left(\right.$ or $\left.p_{n}^{*}\right)$, and $\tilde{d}_{n}(x)$ (or $p_{n}(x)$ ) defined on $x>y_{n}^{1}+d_{n}^{*}$. Also, note that $d_{n}^{*}$ is the same for both systems. The following is the main result on the effect of adding the regular supply mode.

THEOREM 5. After the regular supply is introduced, the optimal value function and optimal policy parameters of the firm satisfy, for $n=1, \ldots, N$,

(i) $v_{n}(x) \geq \hat{v}_{n}(x)$ for all $x$;

(ii) $y_{n}^{1} \leq \hat{y}_{n}^{1}$; (iii) $p_{n}^{*}=\hat{p}_{n}^{*}$;

(iv) $p_{n}(x) \leq \hat{p}_{n}(x)$ for all $x$; and

(v) $\tilde{d}_{n}(x) \geq \hat{d}_{n}(x)$ for all $x$.

We offer the following interpretation of this theorem. Part (i) of Theorem 5 is obvious: when more options become available to the firm, the firm can only do better; thus its maximum profit does not go down. Part (ii) states that the safety-stock level for the expedited supply after adding the second, regular, supply becomes smaller. This is because, after introducing the new supply mode, the firm has another option when needed to raise the inventory level; hence, it can reduce its safety-stock level from the first, expedited, supply. Part (iii) indicates that the optimal list price in the dual-supply system is the same as that in the system with only the expedited supply. However, part (iv) posits that the markdown price in the dual-supply system is lower when the starting inventory level is the same. The last part, (v), is the same as (iv) but stated in terms of optimal average demand.

Proof. Recall that the optimality equation for the dual-supply case is

$$
\begin{aligned}
v_{n}(x) & =\max _{y^{e} \geq x-d, d \in\left[\underline{d}_{n}, \bar{d}_{n}\right]}\left\{\left[R_{n}(d)-c_{n}^{e} d\right]-\left(c_{n}^{e}-c_{n}^{r}\right) y^{e}\right. \\
& \left.-G_{n}\left(y^{e}\right)+\max _{y^{r} \geq y^{e}}\left\{-c_{n}^{r} y^{r}+\alpha \mathbf{E}\left[v_{n-1}\left(y^{r}-\epsilon_{n}\right)\right]\right\}\right\}+c_{n}^{e} x .
\end{aligned}
$$

That $v_{n}(x) \geq \hat{v}_{n}(x)$ can be easily proved using induction, and it is omitted. In the following, we show, by induction, that (ii)-(v) are valid together with $\hat{v}_{n}(y)-v_{n}(y)$ increasing in $y$, that is,

$$
v_{n}^{\prime}(y) \leq \hat{v}_{n}^{\prime}(y)
$$

for $n=1, \ldots, N$.

The result is clearly satisfied when $n=0$ since $v_{0}(x)=\hat{v}_{0}(x)=c_{0}^{e} x$. Now, suppose the result holds for $n-1$, and we proceed to prove it for $n$. For simplicity, let $w_{n}(y)=H_{n}\left(y \vee \tilde{y}_{n}^{2}\right)$. We first show that

$$
c_{n}^{r}+w_{n}^{\prime}(y) \leq \alpha \mathbf{E}\left[\hat{v}_{n-1}^{\prime}\left(y-\epsilon_{n}\right)\right] .
$$

By inductive assumption of Equation (19) for $n-1$, the maximizer $\tilde{y}_{n}^{2}$ of concave function $H_{n}(y)$ is no more than the maximizer of concave function $-c_{n}^{r} y+\alpha \mathbf{E}\left[\hat{v}_{n-1}\left(y-\epsilon_{n}\right)\right]$, denoted by $\hat{y}_{n}^{2}$. If $y \leq \tilde{y}_{n}^{2}$ then the left-hand side of Equation (20) is equal to $c_{n}^{r}$ and, because $y \leq \hat{y}_{n}^{2}$,

$$
\left(-c_{n}^{r} y+\alpha \mathbf{E}\left[\hat{v}_{n-1}\left(y-\epsilon_{n}\right)\right]\right)^{\prime} \geq 0 .
$$

Thus $\alpha \mathbf{E}\left[\hat{v}_{n-1}^{\prime}\left(y-\epsilon_{n}\right)\right] \geq c_{n}^{r}$ and Equation (20) is satisfied. On the other hand, if $y \geq \tilde{y}_{n}^{2}$, then the 
left-hand side of Equation (20) is equal to $\alpha \mathbf{E}\left[v_{n-1}\left(y-\epsilon_{n}\right)\right]$; thus, Equation (20) follows from the inductive assumption.

To complete the proof of Theorem 5, we introduce two functions:

$$
\begin{aligned}
& A_{n}(x, 0)=\max _{y^{e} \geq x}\left\{-c_{n}^{e} y^{e}-G_{n}\left(y^{e}\right)+\left[c_{n}^{r} y^{e}+w_{n}\left(y^{e}\right)\right]\right\}, \\
& A_{n}(x, 1)=\max _{y^{e} \geq x}\left\{-c_{n}^{e} y^{e}-G_{n}\left(y^{e}\right)+\alpha \mathbf{E}\left[\hat{v}_{n-1}\left(y^{e}-\epsilon_{n}\right)\right]\right\},
\end{aligned}
$$

and let $y_{n}^{1}$ and $\hat{y}_{n}^{1}$ be the unconstrained maximizers of the objective functions above, respectively. Then Equation (20) implies $y_{n}^{1} \leq \hat{y}_{n}^{1}$. We further argue that $A_{n}(x, 1)-A_{n}(x, 0)$ is increasing in $x$, or $A_{n}(x, i)$ is supermodular in $(x, i), i=0,1$. To see this, first consider $x \leq y_{n}^{1}$. On this range $A_{n}(x, 1)-A_{n}(x, 0)$ is a constant. If $y_{n}^{1} \leq x \leq \hat{y}_{n}^{1}$, then $A_{n}(x, 0)$ is a constant and $A_{n}(x, 1)$ is increasing; thus $A_{n}(x, 1)-A_{n}(x, 0)$ is also increasing. Finally, if $x \geq \hat{y}_{n}^{1}$, then

$$
A_{n}(x, 1)-A_{n}(x, 0)=\alpha \mathbf{E}\left[\hat{v}_{n-1}\left(x-\epsilon_{n}\right)\right]-\left[c_{n}^{r} x+w_{n}(x)\right]
$$

thus, it follows from Equation (20) that $A_{n}(x, 1)-$ $A_{n}(x, 0)$ is increasing in $x$.

To prove $\hat{v}_{n}(x)-v_{n}(x)$ is increasing in $x$, we further define, for $i=1,0$,

$$
\begin{aligned}
v(x, i)= & \max _{\underline{d}_{n} \leq d \leq \bar{d}_{n}}\left\{\left[R_{n}(d)-c_{n}^{e} d\right]+A_{n}(x-d, i)\right\}+c_{n}^{e} x \\
= & \max _{x-\bar{d}_{n} \leq z \leq x-\underline{d}_{n}}\left\{\left[R_{n}(x-z)-c_{n}^{e}(x-z)\right]+A_{n}(z, i)\right\} \\
& +c_{n}^{e} x,
\end{aligned}
$$

where the second equality follows from the change of variable $z=x-d$. Since both $R_{n}(x-z)-c_{n}^{e}(x-z)$ and $A_{n}(z, i)$ are supermodular in $(x, z, i)$ and the constraint set $\left\{(x, z) \mid x-\bar{d}_{n} \leq z \leq x-\underline{d}_{n}\right\}$ is a lattice, this shows, by application of Lemma 8.4 of Porteus $(2002)$, that $v(x, i)$ is supermodular in $(x, i)$. Finally, note that

$$
v(x, 1)-v_{n}(x, 0)=\hat{v}_{n}(x)-v_{n}(x)
$$

hence, $\hat{v}_{n}(x)-v_{n}(x)$ is also increasing in $x$, completing the proof of Equation (19).

The argument above also shows that $z^{*}(x)=$ $x-d_{n}^{*}(x, i)$ is increasing in $i$. This corresponds to $\tilde{d}_{n}(x) \geq \hat{d}_{n}(x)$, or $p_{n}(x) \leq \hat{p}_{n}(x)$. Finally, $p_{n}^{*}=\hat{p}_{n}^{*}$ is obvious as in both cases, $d_{n}^{*}$ is the maximizer of $R_{n}(d)-c_{n}^{e}(d)$ on $\left[\underline{d}_{n}, \bar{d}_{n}\right]$. The proof of Theorem 5 is thus complete.

REMARK 4. In comparing the ordering policies, we compare the safety-stock levels of the expedited supply before and after the regular supply is introduced. Note that in general we cannot compare the optimal safety-stock level in one model with the safety-stock position in another. That is, we cannot compare the inventory level/position raised by the different supply modes before and after another supply is introduced, and consequently, the overall inventory levels in the two systems cannot be compared. Indeed, the objective for the optimization is the total profit and not the inventory level; since the two supply modes differ in their characteristics, one has shorter lead time but higher cost, holding cost has to weigh in for the inventory optimization decision. Thus, maximizing profit/ minimizing cost does not usually lead to comparison results in the overall inventory levels in the two systems.

\subsection{Impact of Adding an Expedited Supply}

We next consider the case where initially the firm only has the regular supply mode, which has replenishment lead time 1, and we study the impact of introducing an expedited supply that has lead time 0 . Let $\check{v}_{n}(x)$ be the maximum total discounted profit from period $n$ to the end of the planning horizon for the system with only a regular supply, everything else being the same as the original system with dual supply. The optimality equation for this single supply system is

$$
\begin{aligned}
\check{v}_{n}(x)= & \max _{y \geq x, d \in\left[\underline{d}_{n}, \bar{d}_{n}\right]}\left\{R_{n}(d)-c_{n}^{r} y-G_{n}(x-d)\right. \\
& \left.+\alpha \mathbf{E}\left[\check{v}_{n-1}\left(y-d-\epsilon_{n}\right)\right]\right\}+c_{n}^{r} x \\
= & \max _{d \in\left[\underline{d}_{n}, \bar{d}_{n}\right]}\left\{\left[R_{n}(d)-c_{n}^{r} d\right]-G_{n}(x-d)\right. \\
& \left.+\max _{y^{r} \geq x}\left\{-c_{n}^{r}\left(y^{r}-d\right)+\alpha \mathbf{E}\left[\check{v}_{n-1}\left(y^{r}-d-\epsilon_{n}\right)\right]\right\}\right\} \\
& +c_{n}^{r} x \\
= & \max _{d \in\left[\underline{d}_{n},,_{n}\right]}\left\{\left[R_{n}(d)-c_{n}^{r} d\right]-G_{n}(x-d)\right. \\
& \left.+\max _{\check{y}^{r} \geq x-d}\left\{-c_{n}^{r} \check{y}^{r}+\alpha \mathbf{E}\left[\check{v}_{n-1}\left(\check{y}^{r}-\epsilon_{n}\right)\right]\right\}\right\}+c_{n}^{r} x,
\end{aligned}
$$

where $\check{y}^{r}=y^{r}-d$. The optimality equation above leads to the structure of the optimal inventory and pricing decision for the firm, specified in the following result. Since it is a special case of Theorem 4 , its proof is omitted. Let $\check{y}_{n}^{2}=$ $\arg \max _{y}\left\{-c_{n}^{r} y+\alpha \mathbf{E}\left[\check{v}_{n-1}\left(y-\epsilon_{n}\right)\right]\right\}$.

Corollary 1. When the inventory level at the beginning of period $n$ is $x$, the optimal replenishment decision is determined by a critical number $\check{y}_{n}^{2}$ and the optimal average demand $\breve{d}_{n}(x)$, which is an increasing function of starting inventory level $x$ with slope no more than 1 , that is, $0 \leq \breve{d}_{n}(x+1)-\breve{d}_{n}(x) \leq 1$, and the optimal pricing $\check{p}_{n}(x)$ is decreasing in $x$. In particular, when 
$x \leq \check{y}_{n}^{2}+\check{d}_{n}(x)$, the optimal average demand $\check{d}_{n}(x)$ is the maximizer of the single-period concave profit function $R_{n}(d)-c_{n}^{r} d-G_{n}(x-d)$.

It is interesting to note that the optimal price for the model with only a regular supply is no longer of a list-price type, that is, there is no list price $\check{p}_{n}^{*}$. The optimal price $\breve{p}_{n}(x)$ is decreasing in $x$ for all $x$. This is due to the positive lead time. Indeed, when the replenishment lead time is 1 , even if the starting inventory level $x$ at the beginning of period $n$ is very low, after the optimal replenishment decision raises the inventory position to $\check{y}_{n}^{2}+\check{d}_{n}(x)$ the order quantity $\check{y}_{n}^{2}+\check{d}_{n}(x)-x$ will not arrive until the following period, and it is only the on hand inventory, $x$, that is to be used to serve the random demand for the current period. Therefore, no matter how low the inventory level at the beginning of the period might be, the optimal price for this period always depends on $x$. To see the last part of the corollary, note that as long as the inventory level at the beginning of the period is below $\breve{y}_{n}^{2}+\breve{d}_{n}(x)$, the inventory level at the beginning of the next period will be $\check{y}_{n}^{2}-\epsilon_{n}$, which is independent of the decision on average demand $d$. Therefore, the optimal $d$ simply maximizes the profit function for the current period.

As seen from the proof of Theorem 4, the ordering decision for the regular supply is determined by $y_{n}^{2}=\max \left\{\tilde{y}_{n}^{2}, y_{n}^{1}\right\}$, where $y_{n}^{1}$ is the safety-stock level for the expedited supply. Whenever $\tilde{y}_{n}^{2} \leq y_{n}^{1}$, the regular supply is not used in period $n$. Therefore, the optimal ordering decision of the regular supply is determined by $\tilde{y}_{n}^{2}$. In the following theorem, when we compare the ordering characteristics of the regular supply, we compare $\tilde{y}_{n}^{2}$ and $\check{y}_{n}^{2}$.

THEOREM 6. After the expedited supply mode is introduced, the optimal value function and optimal policy parameters of the firm satisfy, for $n=1, \ldots, N$,

(i) $v_{n}(x) \geq \check{v}_{n}(x)$ for all $x$;

(ii) $\tilde{y}_{n}^{2} \leq \check{y}_{n}^{2}$;

(iii) $p_{n}(x) \leq \check{p}_{n}(x)$ for all $x$; and

(iv) $\tilde{d}_{n}(x) \geq \check{d}_{n}(x)$ for all $x$.

Therefore, after the expedited supply is introduced, the profit function of the firm goes up, the safety-stock position goes down, and the optimal selling price drops. This again shows that sourcing diversification benefits both the firm and the customers.
Some preparation is needed to prove Theorem 6 . First, we need the following simple result, which is easy to show.

LEMMA 4. For any concave, differentiable functions $f(x)$ it holds that

$$
f^{\prime}(x) \geq\left(\max _{z \geq x} f(z)\right)^{\prime}
$$

In addition, for convenience we introduce notation

$$
\begin{aligned}
H_{n}(z, 1)= & -G_{n}(z)+\max _{y^{r} \geq z}\left\{-c_{n}^{r} y^{r}+\alpha \mathbf{E}\left[\check{v}_{n-1}\left(y^{r}-\epsilon_{n}\right)\right]\right\} \\
H_{n}(z)= & \left(c_{n}^{r}-c_{n}^{e}\right) z-G_{n}(z) \\
& +\max _{y^{r} \geq z}\left\{-c_{n}^{r} y^{r}+\alpha \mathbf{E}\left[v_{n-1}\left(y^{r}-\epsilon_{n}\right)\right]\right\} \\
H_{n}(x, 0)= & \max _{z \geq x} H_{n}(z) .
\end{aligned}
$$

Then, the optimality equation for the single supply problem can be written as

$$
\begin{aligned}
\check{v}_{n}(x)-c_{n}^{r} x= & \max _{d \in\left[\underline{d}_{n}, \bar{d}_{n}\right]}\left\{\left[R_{n}(d)-c_{n}^{r} d\right]+H_{n}(x-d, 1)\right\} \\
= & \max _{\underline{d}_{n} \leq x-\xi \leq \bar{d}_{n}}\left\{\left[R_{n}(x-\xi)-c_{n}^{r}(x-\xi)\right]\right. \\
& \left.+H_{n}(\xi, 1)\right\},
\end{aligned}
$$

where the second equality follows from a change of variable $\xi=x-d$. We also rewrite the optimality equation for the dual-supply case as

$$
\begin{aligned}
v_{n}(x)-c_{n}^{e} x= & \max _{d \in\left[\underline{d}_{n}, \bar{d}_{n}\right]}\left\{\left[R_{n}(d)-c_{n}^{e} d\right]+H_{n}(x-d, 0)\right\} \\
= & \max _{\underline{d}_{n} \leq x-\xi \leq \bar{d}_{n}}\left\{\left[R_{n}(x-\xi)-c_{n}^{e}(x-\xi)\right]\right. \\
& \left.+H_{n}(\xi, 0)\right\} .
\end{aligned}
$$

Proof of Theorem 6. The first result (i) is intuitive; adding another option does not hurt the firm. Its proof follows easily from induction; the hence it is omitted. Here we only prove (ii), (iii), and (iv).

We will prove inductively that $\check{v}_{n}^{\prime}(x) \geq v_{n}^{\prime}(x)$ for all $x$ and $n$. Note that once this is established, it follows from the definitions of $\tilde{y}_{n}^{2}$ and $\check{y}_{n}^{2}$ that $\tilde{y}_{n}^{2} \leq \check{y}_{n}^{2}$, proving the desired result (ii). This is clearly true for $n=0$. Suppose it has been shown that $\check{v}_{n-1}^{\prime}(x) \geq$ $v_{n-1}^{\prime}(x)$; we proceed to prove it for $n$. From the inductive assumption, we have

$$
\begin{aligned}
& \left(-c_{n}^{r} y^{r}+\alpha \mathbf{E}\left[\check{v}_{n-1}\left(y^{r}-\epsilon_{n}\right)\right]\right)^{\prime} \\
& \geq\left(-c_{n}^{r} y^{r}+\alpha \mathbf{E}\left[v_{n-1}\left(y^{r}-\epsilon_{n}\right)\right]\right)^{\prime}
\end{aligned}
$$


and this implies that for any value $x$, it holds that

$$
\begin{aligned}
& \left(\max _{y^{r} \geq x}\left\{-c_{n}^{r} y^{r}+\alpha \mathbf{E}\left[\check{v}_{n-1}\left(y^{r}-\epsilon_{n}\right)\right]\right\}\right)^{\prime} \\
& \geq\left(\max _{y^{r} \geq x}\left\{-c_{n}^{r} y^{r}+\alpha \mathbf{E}\left[v_{n-1}\left(y^{r}-\epsilon_{n}\right)\right]\right\}\right)^{\prime} .
\end{aligned}
$$

Therefore, we obtain
For fixed $x$, let

$$
\begin{aligned}
& \check{\xi}_{n}^{*}(x)=\arg \max _{\underline{d}_{n} \leq x-\xi \leq \bar{d}_{n}} g(x, \xi, 1) \\
& \xi_{n}^{*}(x)=\arg \max _{\underline{d}_{n} \leq x-\xi \leq \bar{d}_{n}} g(x, \xi, 0) .
\end{aligned}
$$

Then, by the supermodularity result we conclude that both $\check{\xi}_{n}^{*}(x)$ and $\xi_{n}^{*}(x)$ are increasing in $x$, and

$$
\begin{aligned}
c_{n}^{r}-c_{n}^{e}+H_{n}^{\prime}(x, 1) & =\left(\left(c_{n}^{r}-x_{n}^{e}\right) x-G_{n}(x)+\max _{y^{r} \geq x}\left\{-c_{n}^{r} y^{r}+\alpha \mathbf{E}\left[\check{v}_{n-1}\left(y^{r}-\epsilon_{n}\right)\right]\right\}\right)^{\prime} \\
& \left.\geq\left(\left(c_{n}^{r}-c_{n}^{e}\right) x-G_{n}(x)+\max _{y^{r} \geq x}\left\{-c_{n}^{r} y^{r}+\alpha \mathbf{E}\left[v_{n-1}\left(y^{r}-\epsilon_{n}\right)\right)\right]\right\}\right)^{\prime} \\
& \geq\left(\max _{z \geq x}\left\{\left(c_{n}^{r}-c_{n}^{e}\right) z-G_{n}(z)+\max _{y^{r} \geq z}\left\{-c_{n}^{r} y^{r}+\alpha \mathbf{E}\left[v_{n-1}\left(y^{r}-\epsilon_{n}\right)\right]\right\}\right\}\right)^{\prime} \\
& =H_{n}^{\prime}(x, 0),
\end{aligned}
$$

where the last inequality follows from Lemma 4 . Then, we apply the same idea in proving Theorem 5. Define $g(x, \xi, i)$ on $-\infty<x, \xi<\infty$ and $i=0,1$ by

$$
\begin{aligned}
& g(x, \xi, 1)=R_{n}(x-\xi)+c_{n}^{r} \xi+H_{n}(\xi, 1) \\
& g(x, \xi, 0)=R_{n}(x-\xi)+c_{n}^{e} \xi+H_{n}(\xi, 0) .
\end{aligned}
$$

By the concavity of $R_{n}(\cdot), g(x, \xi, i)$ is supermodular in $(x, \xi)$, and because

$$
g(x, \xi, 1)-g(x, \xi, 0)=H_{n}(\xi, 1)-H_{n}(\xi, 0)-\left(c_{n}^{e}-c_{n}^{r}\right) \xi
$$

is increasing in $\xi$ from Equation (24) and independent of $x$, we conclude that $g$ is supermodular in $(x$, i) and $(\xi, i)$. This proves that $g(x, \xi, i)$ is supermodular in $(x, \xi, i)$. Since the constraint set $\underline{d}_{n} \leq x-\xi \leq \bar{d}_{n}$ is a sublattice of $(x, \xi, i)$, we apply Lemma 8.4 of Porteus (2002) to obtain that

$$
g(x, i)=\max _{\underline{d}_{n} \leq x-\xi \leq \bar{d}_{n}} g(x, \xi, i)
$$

is supermodular in $(x, i)$. Noting from Equations (20) and (21) that

$$
\check{v}_{n}(x)=\max _{\underline{d}_{n} \leq x-\xi \leq \bar{d}_{n}} g(x, \xi, 1), v_{n}(x)=\max _{\underline{d}_{n} \leq x-\xi \leq \bar{d}_{n}} g(x, \xi, 0),
$$

we conclude that

$$
g(x, 1)-g(x, 0)=\check{v}_{n}(x)-v_{n}(x)
$$

is increasing in $x$, or $\check{v}_{n}^{\prime}(x) \geq v_{n}^{\prime}(x)$. This proves that $\check{v}_{n}^{\prime}(x) \geq v_{n}^{\prime}(x)$ holds for all $n$. that $\xi_{n}^{*}(x) \leq \check{\xi}_{n}^{*}(x)$ for all $x$. As $\check{\xi}_{n}^{*}(x)_{\sim}=x-\check{d}_{n}(x)$ and $\xi_{n}^{*}(x)=x-\tilde{d}_{n}(x)$, this implies $\tilde{d}_{n}(x) \geq \tilde{d}_{n}(x)$, proving (iii). Hence, the optimal prices, $p_{n}(x)$ and $\check{p}_{n}(x)$, are both decreasing in the inventory level $x$ and they satisfy $p_{n}(x) \leq \check{p}_{n}(x)$ for all $x$, or (iv). That is, the optimal price decreases after the expedited supply is introduced under a same starting inventory level. The proof of Theorem 6 is complete.

\section{Numerical Study}

In this section, we aim at studying the following questions numerically. First, what is the respective value to the firm of having dual supply options and adopting dynamic pricing? Second, which strategic tool brings more value to the firm?

We first consider a system with planning horizon $N=5$. Suppose demand function $D(p)=a-\gamma p+\epsilon$ in which $\epsilon$ is Negative Binomial with mean $8 ; a=100$ and $\gamma=2$. This is an additive model with $d_{n}(p)=a-\gamma p+\mathbf{E}[\epsilon]$ and $\epsilon_{n}=\epsilon-\mathbf{E}[\epsilon]$. The unit regular ordering $\operatorname{cost}$ is $c_{n}^{r}=2$; the last period salvage value/penalty cost is $c_{0}^{e}=2$; the inventory holding and demand backlogging cost is $L(x)=h x^{+}+b x^{-}$ with $h=2$ and $b=20$ when the ending inventory level is $x$. The discount factor $\alpha=0.95$. Other system parameters are selected from the following sets:

$$
c_{n}^{e}=\{4,8,16\}, \quad \operatorname{var}(\epsilon)=\{10,13.33,20,40\} .
$$

We will discuss the value of supply diversification in both dynamic and static pricing settings, while for the value of dynamic pricing, we consider the case with single and dual supplies. So we first specify how the static price is selected. When the expedited supply 
is available (either the firm uses both supplies or the expedited supply only), the static price $p^{s}$ is the one that maximizes the single-period profit function, that is,

$$
\begin{aligned}
\left(p^{s}, y^{s}\right)= & \arg \max _{p, y}\left\{-(1-\alpha) c_{n}^{e}+\left(p-\alpha c_{n-1}^{e}\right) d(p)\right. \\
& \left.-G_{n}(y, p)\right\} .
\end{aligned}
$$

A similar static pricing policy is used in the numerical study of Federgruen and Heching (1999). When only the regular supply is available, we choose the static price $p^{s}$ as the one that maximizes the expected revenue $p d(p)$ because, in this case the ordering lead time is one period, and we want to avoid a statedependent static price. Denote the corresponding value function under the static pricing strategy by $v_{n}^{s}(x)$.

To assess the benefit of supply diversification, we compare the profit of the system with dual supply vis-à-vis the one with a single sourcing when the firm uses either dynamic pricing or a static pricing strategy. For the systems with dynamic pricing, we define

$$
V O D=\text { average }_{x \in[-10,60]}\left\{\frac{v_{N}(x)-A}{v_{N}(x)} \times 100 \%\right\},
$$

where $v_{N}(x)$ is the optimal profit under dual sourcing while $A=\hat{v}_{N}(x)$ or $\check{v}_{N}(x)$ is the optimal profit with only the expedited (regular) supply. Similarly, for the case with static pricing, the value of supply diversification $V O D^{s}$ is defined as

$$
V O D^{s}=\text { average }_{x \in[-10,60]}\left\{\frac{v_{N}^{s}(x)-B}{v_{N}^{s}(x)} \times 100 \%\right\},
$$

in which $B=\hat{v}_{N}^{s}(x)$ or $\check{v}_{N}^{s}(x)$, and $v_{N}^{s}(x)$ uses the same static price as that used in computing $\hat{v}_{N}^{s}(x)$ or $\check{v}_{N}^{s}(x)$.

Table 1 presents $V O D$ and $V O D^{s}$ under different demand variances and unit expedited costs. For ease of reference, we use $V O D_{j}$ and $V O D_{j}^{s}$ to denote the corresponding value when only supply $j$ is available, $j=r, e$. First, note that when the unit expedited cost increases, $V O D_{r}$ and $V O D_{r}^{s}$ decrease whereas $V O D_{e}$ and $V O D_{e}^{s}$ increase. This is intuitive, as when compared to the case with only regular supply, the expedited supply becomes less important when its cost gets higher, whereas when compared to the case with only expedited supply, the value of having a regular supply would go up when the expedited supply becomes more costly. Meanwhile, we find the value of supply diversification, in both dynamic and static pricing settings, is rather robust to the change of the variance of $\varepsilon$.

We then examine the value of dynamic pricing, which is quantified by

$$
V O P=\text { average }_{x \in[-10,60]}\left\{\frac{v_{N}(x)-v_{N}^{s}(x)}{v_{N}(x)} \times 100 \%\right\},
$$

and, analogously, when there is only the expedited (resp., regular) supply available, the corresponding $V O P$ is defined by replacing $v_{N}(x)$ with $\hat{v}_{N}(x)$ (resp., $\check{v}_{N}(x)$ ) and $v_{N}^{s}(x)$ with $\hat{v}_{N}^{s}(x)$ (resp., $\check{v}_{N}^{s}(x)$ ) and we denote it by $V O P_{e}$ (resp., $V O P_{r}$ ).

The results are reported in Table 2. It can be seen that under supply diversification, the value of dynamic pricing is rather small when the unit cost of expedited order is relatively low (VOP is $<2 \%$ even when $c_{n}^{e}=8$, four times the regular unit cost), while it increases dramatically when the unit expedited cost reaches 16. This interesting finding can be explained as follows. When the expedited supply is inexpensive, the firm enjoys supply flexibility, which can be used to mitigate the adverse effect caused by uncertainty in random demand, and it limits the value of dynamic pricing. In single sourcing setting, when only the regular supply is available, $V O P_{r}$ is around $3.9 \%$ whereas $V O P_{e}$ is very minimum when only the expedited supply is available. The latter phenomenon is in part because when the starting inventory level falls in the region $[-10,60]$, the optimal price is the same as the static one in most of the cases. However, we find that dynamic pricing brings higher value to the firm when the starting inventory level is more than 60 . Further-

\begin{tabular}{|c|c|c|c|c|c|c|c|c|c|c|c|c|}
\hline \multirow[b]{3}{*}{$\underline{\operatorname{Var}[\varepsilon]}$} & \multicolumn{6}{|c|}{ Dynamic pricing } & \multicolumn{6}{|c|}{ Static pricing } \\
\hline & \multicolumn{3}{|c|}{$V O D_{r}$} & \multicolumn{3}{|c|}{$V O D_{e}$} & \multicolumn{3}{|c|}{$V O D_{r}^{S}$} & \multicolumn{3}{|c|}{$V O D_{e}^{S}$} \\
\hline & $c_{n}^{e}=4$ & $c_{n}^{e}=8$ & $c_{n}^{e}=16$ & $c_{n}^{e}=4$ & $c_{n}^{e}=8$ & $c_{n}^{e}=16$ & $c_{n}^{e}=4$ & $c_{n}^{e}=8$ & $c_{n}^{e}=16$ & $c_{n}^{e}=4$ & $c_{n}^{e}=8$ & $c_{n}^{e}=16$ \\
\hline 10 & 5.56 & 3.94 & 1.37 & 5.50 & 17.01 & 37.92 & 8.69 & 6.75 & 2.91 & 5.36 & 16.02 & 33.63 \\
\hline 13.33 & 5.55 & 3.90 & 1.34 & 5.50 & 17.03 & 37.99 & 8.69 & 6.72 & 2.87 & 5.36 & 16.04 & 33.69 \\
\hline 20 & 5.53 & 3.85 & 1.30 & 5.49 & 17.06 & 38.11 & 8.69 & 6.67 & 2.83 & 5.35 & 16.06 & 33.79 \\
\hline 40 & 5.52 & 3.74 & 1.23 & 5.47 & 17.13 & 38.37 & 8.70 & 6.58 & 2.75 & 5.34 & 16.13 & 34.03 \\
\hline
\end{tabular}
more, the value of dynamic pricing is also rather insensitive to the variance of $\varepsilon$, in large part because the demand function is additive. We depict the

Table 1 Value of Supply Diversification 
Table 2 Value of Dynamic Pricing

\begin{tabular}{|c|c|c|c|c|c|c|c|}
\hline \multirow[b]{3}{*}{$\underline{\operatorname{Var}[\varepsilon]}$} & \multicolumn{3}{|c|}{ Dual sourcing } & \multicolumn{4}{|c|}{ Single sourcing } \\
\hline & & VOP & & \multirow{2}{*}{$\begin{array}{r}V O P_{r} \\
C_{n}^{r}=2\end{array}$} & \multicolumn{3}{|c|}{$V O P_{e}$} \\
\hline & $c_{n}^{e}=4$ & $c_{n}^{e}=8$ & $c_{n}^{e}=16$ & & $c_{n}^{e}=4$ & $c_{n}^{e}=8$ & $c_{n}^{e}=16$ \\
\hline 10 & 0.15 & 1.19 & 6.47 & 3.84 & 0.00 & 0.01 & 0.02 \\
\hline 13.33 & 0.15 & 1.19 & 6.48 & 3.85 & 0.00 & 0.00 & 0.02 \\
\hline 20 & 0.15 & 1.19 & 6.52 & 3.87 & 0.00 & 0.00 & 0.01 \\
\hline 40 & 0.14 & 1.19 & 6.58 & 3.90 & 0.00 & 0.00 & 0.01 \\
\hline
\end{tabular}

Figure 1 Comparison of Value Functions

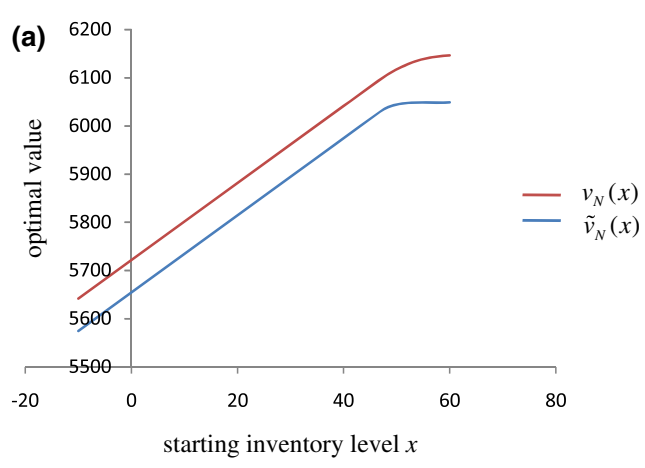

Table 3 Impact of Holding Cost $h$ on VOD and VOP

\begin{tabular}{llllllll}
\hline$h$ & $V O D_{r}$ & $V O D_{e}$ & $V O D_{r}^{s}$ & $V O D_{e}^{s}$ & $V O P$ & $V O P_{r}$ & $V O P_{e}$ \\
\hline 2 & 3.94 & 17.01 & 6.75 & 16.02 & 1.19 & 3.84 & 0.01 \\
4 & 3.89 & 17.07 & 6.79 & 15.97 & 1.29 & 3.82 & 0.01 \\
6 & 3.90 & 17.10 & 6.84 & 15.94 & 1.42 & 3.95 & 0.04 \\
\hline
\end{tabular}

optimal profit function under dynamic pricing and static pricing in Figure 1a and the optimal profit under dual sourcing and single sourcing in Figure 1b.

To see how inventory holding cost $h$ affects the values of supply diversification and dynamic pricing, we conduct additional numerical experiments by altering the values of $h$ from 2 to 6 with a step size 2 while letting $c_{n}^{e}=8$ and $\operatorname{Var}[\varepsilon]=10$. The results are presented in Table 3. We can see that $h$ has a minor impact on those values.

From the results in Tables $1-3$, we find that in general, supply diversification provides the firm with more benefit than dynamic pricing, except when the expedited supply is very costly $\left(c_{n}^{e}=16\right)$, and in that case $V O D_{r}^{s}$ is less than $V O P_{r}$, implying that dynamic pricing has bigger impact. In this regard, these two strategic tools seem to be substitutes for each other. Suppose, for example, the firm is currently using dual supply modes and adopting a dynamic pricing strategy and is considering simplifying its operation by either reducing its supply base or implementing a simple static pricing strategy. Then, the results of $V O D_{r}, V O D_{e}$, and $V O P$ suggest that when $c_{n}^{e}$ is

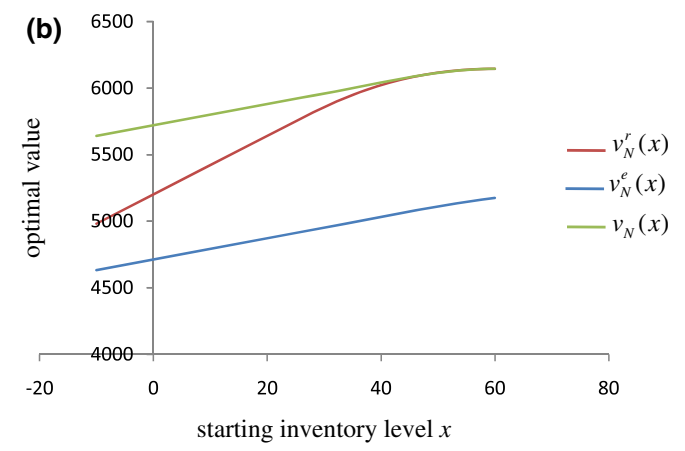

relatively low, instituting a static price strategy will not hurt much of the firm's profit and, hence, is the recommended action to take, while when $c_{n}^{e}$ is relatively high, then it is better that the firm simply remove the expedited supply mode from its supply base.

We finally study the comparative static properties of optimal policy parameters. In Figure 2a, we illustrate the trajectory of the optimal price for the systems with (a) single sourcing with regular supply, (b) single sourcing with expedited supply, and (c) dual sourcing. It is clear that the optimal price decreases with the starting inventory level in all three cases. Furthermore, the optimal selling price for the dual supply system is always the lowest, which is consistent with our theoretical results that sourcing diversification is beneficial to the customers. The comparison of the optimal selling prices between the two single sourcing systems, however, depends on the starting inventory level: the optimal selling price for the system with single regular supply is the highest when the starting inventory level is low, and this may be attributed to the longer inventory replenishment time. However, when the inventory level is high, the system with the single expedited supply has the highest selling price, and the intuition behind this is that the firm does not want to get rid of its inventory too quickly because of its higher unit ordering cost.

Figure $2 \mathrm{~b}$ illustrates the optimal inventory level/ position after replenishment decisions in the three 
Figure 2 Optimal Selling Prices and Order-up-to Levels

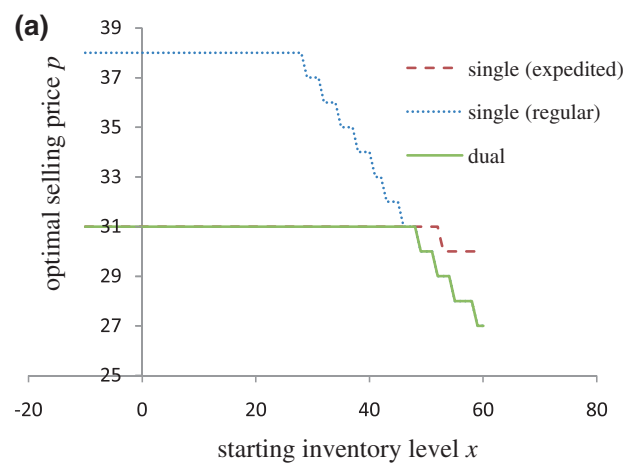

Table 4 VOD and VOP for $T=20$

\begin{tabular}{lccccccc}
\hline & \multicolumn{4}{c}{ VOD } & & \multicolumn{3}{c}{ VOP } \\
\cline { 2 - 4 } \cline { 6 - 7 } $\operatorname{Var}[\varepsilon]$ & $c_{n}^{e}=4$ & $c_{n}^{e}=8$ & $c_{n}^{e}=16$ & & $c_{n}^{e}=4$ & $c_{n}^{e}=8$ & $c_{n}^{e}=16$ \\
\hline 10 & 1.96 & 1.35 & 0.45 & & 0.24 & 0.44 & 0.94 \\
13.33 & 1.97 & 1.34 & 0.44 & & 0.24 & 0.44 & 0.95 \\
20 & 1.98 & 1.32 & 0.43 & & 0.24 & 0.45 & 0.98 \\
40 & 2.03 & 1.30 & 0.41 & & 0.24 & 0.46 & 1.02 \\
\hline
\end{tabular}

systems, similar to those in Figure 2a. In the figure, $s^{e}$ (= 47) pinpoints the optimal expedited order-up-to level in the dual sourcing system, which is lower than that $(=50)$ of the system with single expedited supply. This is due to the fact that the dual sourcing system has another option to order from the regular supply, and it echoes the result in Theorem 5. Nevertheless, the dual sourcing system keeps a higher regular order-up-to level than the system with only regular supply, especially when $x<s_{n}^{e}$. Note that the $S^{r}(x)$ values for the two systems are actually quite close. As in the dual sourcing system, the firm can increase its inventory level to $s_{n}^{e}$ immediately and then charge a lower price, and it would keep a higher regular order-up-to level.

We end this section by reporting $V O D$ and $V O P$ values for a system with a longer planning horizon, $T=20$, in Table 4 . The other parameters are the same as those of the preceding examples. It can be seen that the general messages conveyed in the previous examples carry over to this case, and the values of these two strategic tools, for example, supply diversification and dynamic pricing, do not vanish with a long planning horizon.

\section{Concluding Remarks}

In this paper we study the dynamic pricing and inventory control in a periodic-review inventory system with dual supply modes. The lead times for the two supply modes are 0 and 1 , respectively. We show that the optimal inventory policy is of a base-stock

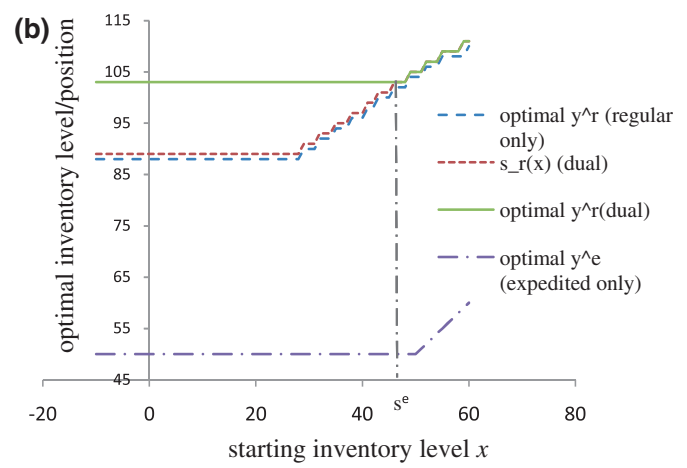

type, and the optimal pricing strategy is list price with markdowns. We also analyze the effect of supply diversification and prove that supply diversification benefits both the firm and its customers. Our numerical results further show that supply diversification brings the firm more value than dynamic pricing and these two strategies seem to be substitutes for each other.

One critical assumption in our model is that the lead times of the two supply modes are 0 and 1 , respectively. When the selling price is exogenous, the optimal inventory control problem with dual-supply and arbitrary lead times has been studied in Whittmore and Saunders (1977), who show that the optimal policy for such a system is quite complicated; its policy control parameters depend on the state of the system. When there is only a single supply but arbitrary lead time, the joint optimization problem of inventory and pricing is studied in Pang et al. (2012). Both the optimal inventory control policy and the optimal pricing strategy are shown to be state dependent, that is, the policy control parameters depend on the state of the system. Thus, if the lead times of the two supply modes are arbitrary, it is conceivable that the operational strategies for the model discussed in this study can only be more complicated than those in Whittmore and Saunders (1977) and Pang et al. (2012). Some preliminary discussion is given below.

Suppose the lead times for the two supply modes are 0 and $L$, respectively, where $L \geq 1$ is an arbitrary integer. Let $x_{0}$ be the inventory level at the beginning of a period and $x_{1}, x_{2}, \ldots, x_{L-1}$ the orders that are to be delivered one period from now, two periods from now, and $L-1$ periods from now. The state of the system is $\left(x_{0}, x_{1}, \ldots, x_{L-1}\right)$. To analyze the structure of the optimal inventory and pricing policy, we reformulate the problem as follows: define

$$
y_{i}=\sum_{k=i}^{L-1} x_{k}, \quad i=0,1, \ldots, L-1
$$


The transformed state of the system is $\mathbf{y}=\left(y_{0}, y_{1}, \ldots, y_{L-1}\right)$, with state space

$$
y_{1} \geq y_{2} \geq \cdots \geq y_{L-1}
$$

Using $L^{\natural}$-convexity and lattice analysis, our preliminary analysis shows that the optimal replenishment decisions and the optimal price depend on the state $\mathbf{y}$ in some complicated but monotone manner. This will be investigated in a future study.

\section{Acknowledgments}

The authors are grateful to the senior editor and the two referees for their insightful comments and suggestions, which have helped us greatly improve the quality and exposition of this paper. In particular, section 5 was added based on the suggestions from the review team. The first author is partially supported by Hong Kong GRF fund CUHK-419010, and the second author is partially supported by NSF under CMMI-0927631 and CMMI-1131249.

\section{References}

Allon, G., J. A. Van Mieghem. 2010. Global dual sourcing: Tailored base-surge allocation to near- and offshore production. Manage. Sci., 56(1): 110-124.

Allon, G., A. Zeevi. 2011. A note on the relationship among price, capacity and inventory decisions in a make-to-stock system. Prod. Oper. Manag. 20(1): 143-151.

Barankin, E. W. 1961. A delivery-lag inventory model with an emergency provision (the one-period case). Naval Res. Logist. Quart. 8(3): 258-311.

Bertsekas, D. P. 2007. Dynamic Programming and Optimal Control. Athena Scientific, Nashua, NH.

Chen, X., D. Simchi-Levi. 2004a. Coordinating inventory control and pricing strategies with random demand and fixed ordering cost: The finite horizon case. Oper. Res. 52(6): 887896.

Chen, X., D. Simchi-Levi, 2004b. Coordinating inventory control and pricing strategies with random demand and fixed ordering cost: The infinite horizon case. Math. Oper. Res. 29(3): 698-723.
Chen, X., D. Simchi-Levi. 2010. Pricing and inventory management. R. Philips, O. Ozalp, eds. Handbook of Pricing, Oxford University Press, pp. 785-822.

Daniel, K. 1963. A delivery-lag inventory model with emergency. H. Scarf, D. Gilford, M. Shelly, eds. Multistage Inventory Models and Techniques. Stanford University Press, Stanford, CA. pp. 32-46.

Federgruen, A., A. Heching. 1999. Combined pricing and inventory control under uncertainty. Oper.Res. 47(3): 454-475.

Feng, Q., G. Gallego, S. P. Sethi, H. Yan H. Zhang. 2003. A periodic review inventory model with three consecutive delivery modes and forecast updates. Working paper. University of Texas at Dallas, Richardson, TX.

Feng, Q., G. Gallego, S. P. Sethi, H. Yan, H. Zhang. 2004. Optimality and nonoptimality of the base-stock policy in inventory problems with multiple delivery modes. Working paper. University of Texas at Dallas, Richardson, TX.

Feng, Y., F. Chen. 2003. Pricing and inventory control for a periodic-review system: Optimality and optimization of $(s, S, p)$ policy. Working paper. The Chinese University of Hong Kong, Shatin, N.T. Hong Kong.

Fukuda, Y. 1964. Optimal policies for the inventory problem with negotiable leadtime. Manage. Sci. 10(4): 690-708.

Heyman, D. P., M. J. Sobel. 1984. Stochastic Models in Operations Research, Volume II. McGraw-Hill, New York.

Mills, E. S. 1959. Uncertainty and price theory. Quart. J. Econ. 73(1): 116-130.

Pang, Z., F. Y. Chen, Y. Feng. 2012. A note on the structure of joint inventory-pricing control with leadtimes. Oper. Res. 60(3): 581-587.

Porteus, E. L. 2002. Foundations of Stochastic Inventory Theory. Stanford University Press, Stanford, CA.

Scheller-Wolf, A., G. J. van Houtum, S. Veeraraghavan 2003. Inventory models with expedited ordering: Single index policies (unabridged version). Working paper. Carnegie Mellon University, Pittsburgh, PA.

Topkis, D. M. 1998. Supermodularity and Complementarity. Princeton University Press, Princeton, NJ.

Veeraraghavan, S., A. Scheller-Wolf. 2008. Now or later: A simple policy for effective dual sourcing in capacitated systems. Oper. Res. 56(4): 850-864.

Whitin, T. T. 1955. Inventory control and price theory. Manage. Sci. 2(1): 61-80.

Whittmore, A. S., S. C. Saunders. 1977. Optimal inventory under stochastic demand with two supply options. SIAM J. Appl. Math. 32(2): 293-305. 